\title{
Higher-order linking forms for knots
}

\author{
Constance Leidy
}

\begin{abstract}
We construct examples of knots that have isomorphic $n$ th-order Alexander modules, but non-isomorphic $n$ th-order linking forms, showing that the linking forms provide more information than the modules alone. This generalizes work of Trotter [T], who found examples of knots that have isomorphic classical Alexander modules, but non-isomorphic classical Blanchfield linking forms.
\end{abstract}

Mathematics Subject Classification (2000). 57M25.

Keywords. Blanchfield form, Alexander module, knot group, derived series, localization of rings.

\section{Introduction}

In 1973, Trotter [T] found examples of knots that have isomorphic classical Alexander modules, but non-isomorphic classical Blanchfield linking forms. Recently, T. Cochran $[C]$ defined higher-order Alexander modules, $\mathcal{A}_{n}(K)$, of a knot, $K$, and higher-order linking forms, $\mathcal{B} \ell_{n}(K)$, which are linking forms defined on $\mathcal{A}_{n}(K)$. When $n=0$, these invariants are just the classical Alexander module and Blanchfield linking form. The question was posed in $[\mathrm{C}]$ whether Trotter's result generalized to the higher-order invariants. We show that it does. The following is our main theorem.

Main Theorem. For each $n \geq 0$, there exist knots $K_{0}$ and $K_{1}$ such that $\mathcal{A}_{i}\left(K_{0}\right) \cong$ $\mathcal{A}_{i}\left(K_{1}\right)$ for $0 \leq i \leq n$ and $\mathscr{B} \ell_{i}\left(K_{0}\right) \cong \mathscr{B} \ell_{i}\left(K_{1}\right)$ for $0 \leq i<n$, but $\mathscr{B}_{n}\left(K_{0}\right) \nsucceq$ $\mathscr{B} \ell_{n}\left(K_{1}\right)$.

When $n=1$, a particular example of the main theorem is the pair of knots depicted below. The construction of them will be explained later in this paper.

We shall work with classical, oriented knots in the PL category. We now review some notions of classical knot theory. We refer the reader to [G], [Li], and [Ro] as knot theory resources. Recall that by Alexander duality, the $p$-th reduced homology of the exterior of the knot is trivial except when $p=1$, in which case it is $\mathbb{Z}$, 

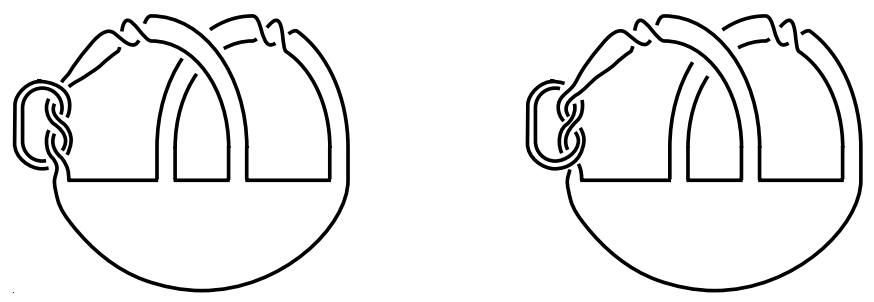

generated by the meridian. It follows that $\frac{G}{G^{\prime}} \cong \mathbb{Z}$, where $G$ is the fundamental group of the exterior. Hence, we can take the infinite cyclic cover of the exterior. The classical Alexander module of a knot is defined to be the first homology of this infinite cyclic cover of the exterior of the knot, considered as a $\mathbb{Z}\left[t, t^{-1}\right]$-module. Here the module structure results from the action $x * t=\mu^{-1} x \mu$, where $\mu$ is the meridian of the knot. Furthermore, since the fundamental group of the infinite cyclic cover is the commutator subgroup, $G^{\prime}$, it follows that the Alexander module is simply $\frac{G^{\prime}}{G^{\prime \prime}}$ considered as a right $\mathbb{Z}\left[\frac{G}{G^{\prime}}\right]$-module.

A Seifert surface for a knot, $K$, is a connected, bicollared, compact surface in $S^{3}$ whose boundary is $K$. For a choice of Seifert surface, $F$, and bicollar, the Seifert form on $H_{1}(F)$ is defined to be the linking number of $x$ with $y^{+}$, for any $x, y \in H_{1}(F)$, where $y^{+}$denotes a pushoff of $y$ in the positive direction of the bicollar of $F$. A Seifert matrix $V$ is the matrix representing the Seifert form with respect to a choice of basis for $H_{1}(F)$. For any Seifert matrix $V$, recall that $V-t V^{T}$ presents the Alexander module and $\operatorname{det}\left(V-V^{T}\right)=1 \neq 0$. It follows that the Alexander module is a torsion module. That is, for any element of the Alexander module, $x$, there is a non-zero element, $p(t)$, of $\mathbb{Z}\left[t, t^{-1}\right]$ such that $x * p(t)=0$.

Since the Alexander module is a torsion module, it is possible to define a linking form on the Alexander module, known classically as the Blanchfield linking form. Let $x$ and $y$ be any two elements of the Alexander module. Then as above, there is a non-zero element of $\mathbb{Z}\left[t, t^{-1}\right], p(t)$, such that $x * p(t)=0$. Therefore there is a 2 -chain, $\alpha$, in the infinite cyclic cover of the exterior whose boundary is $x * p(t)$. We define the Blanchfield linking form of $x$ and $y$ to be

$$
\mathscr{B} \ell(x, y)=\sum_{i=-\infty}^{\infty} \frac{1}{p\left(t^{-1}\right)} \lambda\left(\alpha, y * t^{i}\right) t^{-i} \quad\left(\bmod \mathbb{Z}\left[t, t^{-1}\right]\right),
$$

where $\lambda$ is the ordinary intersection form. Notice that the Blanchfield linking form takes values in $\mathbb{Q}(t) / \mathbb{Z}\left[t, t^{-1}\right]$.

In order to motivate our main theorem, we recall some results about the Blanchfield linking form. C. Kearton $[\mathrm{K}]$ and H. F. Trotter $[\mathrm{T}]$ each proved the following theorem.

Theorem 1.1. If $V$ is a Seifert matrix for a knot $K$, then $(1-t)\left[V-t V^{T}\right]^{-1}$ represents the Blanchfield linking form for $K$. 
Recall that $\mathrm{S}$-equivalence of matrices is the equivalence relation generated by integral congruence and column enlargements. Here $V$ is integrally congruent to $P^{T} V P$, where $P$ is an integral matrix with $\operatorname{det} P= \pm 1$, and a column enlargement of $V$ is the following:

$$
\left(\begin{array}{ccc}
V & u^{T} & 0 \\
v & x & 1 \\
0 & 0 & 0
\end{array}\right) .
$$

Here $x$ is an integer and $u$ and $v$ are column vectors. Furthermore, two knots are $\mathrm{S}$-equivalent if they have $\mathrm{S}$-equivalent Seifert matrices.

Proposition 1.2 ([T], p. 179; [K], p. 142). Two knots have isomorphic Blanchfield linking forms if and only if they are S-equivalent.

The question arises: Do there exist knots with isomorphic Alexander modules, but non-isomorphic Blanchfield forms? By Proposition 1.2, it suffices to find examples of knots with isomorphic Alexander modules that are not S-equivalent. Furthermore, since the ordinary signature of a knot is an S-equivalence invariant, we have reduced the problem to finding two knots with isomorphic Alexander modules, but with different signatures.

Given any knot, $K$, let $-K=r \bar{K}$ denote the reverse of the mirror-image of $K$. This is also the inverse of $K$ in the knot concordance group.

Proposition 1.3. If $K$ is a knot such that the ordinary signature of $K$ is non-zero, then $K$ and $-K$ have isomorphic Alexander modules, but non-isomorphic Blanchfield linking forms.

Proof. If $V$ is a Seifert matrix for $K$, then $-V^{T}$ is a Seifert matrix for its mirrorimage, $\bar{K}$, and $V^{T}$ is a Seifert matrix for its reverse, $r K$. Therefore the Seifert matrix for $-K$ is $-V$. Since $V-t V^{T}$ and $-V+t V^{T}$ present isomorphic modules, $K$ and $-K$ have isomorphic Alexander modules. However, if the signature of $K$ is non-zero, then the signature of $-K$ is not equal to the signature of $K$. Hence, $K$ and $-K$ are not $S$-equivalent, and therefore have non-isomorphic Blanchfield linking forms.

We note that the examples that Trotter provided in [T] were found using different methods than those presented here.

\section{Higher-order Alexander modules and linking forms}

Let us recall some of the definitions and results from [C] and [COT1]. Given a knot $K$, let $E(K)$ denote the exterior of $K, S^{3} \backslash \operatorname{nbhd}(K)$, and let $G=\pi_{1}(E(K)$ ). 
Recall that the derived series of a group $H$ is defined recursively by $H^{(0)}=H$ and $H^{(n+1)}=\left[H^{(n)}, H^{(n)}\right]$, for $n \geq 1$. We will use $\Gamma_{n}$ to denote the quotient group $\frac{G}{G^{(n+1)}}$. Then we have the coefficient system defined by the homomorphism $G \rightarrow \Gamma_{n}$.

Definition 2.1. For $n \geq 0$, the $n$th higher-order Alexander module of a knot $K$ is

$$
\mathcal{A}_{n}(K) \equiv H_{1}\left(E(K) ; \mathbb{Z} \Gamma_{n}\right) .
$$

Therefore the $n$th higher-order Alexander module is the first (integral) homology group of the covering space of the knot exterior corresponding to $G^{(n+1)}$, considered as a right $\mathbb{Z} \Gamma_{n}$-module. This is the same as $\frac{G^{(n+1)}}{G^{(n+2)}}$ as a right $\mathbb{Z} \Gamma_{n}$-module. (Notice that we are working with the right $\mathbb{Z} \Gamma_{n}$-module structure on the chain groups given by $\alpha * g=g \alpha g^{-1}$.)

As in the classical case, the higher-order Alexander modules of a knot are torsion modules ([C], Prop. 3.10). Therefore, it is possible to define symmetric linking forms on the higher-order Alexander modules. Before giving the careful algebraic definition, we describe the geometric idea of the linking forms. Given any element $x \in \mathcal{A}_{n}(K)$, there is some $\gamma \in \mathbb{Z} \Gamma_{n}$ such that $x \cdot \gamma=0$. Therefore $x \cdot \gamma$ can be represented as the boundary of a 2-chain, $\alpha \in C_{2}\left(X ; \mathbb{Z} \Gamma_{n}\right)$. For any $y \in \mathcal{A}_{n}(K)$, define $B \ell_{n}(x, y) \equiv \bar{\gamma}^{-1} \cdot \lambda_{n}(\alpha, y)$, where $\lambda_{n}$ denotes the equivariant intersection pairing on $E(K)$ with coefficients in $\mathbb{Z} \Gamma_{n}$. Here $\bar{\gamma}$ is the image of $\gamma$ under the group ring involution defined on the group ring $\mathbb{Z} \Gamma_{n}$ by $\overline{\left(\sum n_{i} g_{i}\right)}=\sum n_{i} g_{i}^{-1}$ (see [P, p. 5]). Since we are working with the right module structure, the equivariant intersection pairing is defined as

$$
\lambda_{n}(\alpha, y)=\sum_{g \in \Gamma_{n}} \lambda(\alpha,(y * g)) \cdot g^{-1},
$$

where $\lambda$ denotes the ordinary intersection form.

In [COT1, Prop. 3.2], it is shown that $\mathbb{Z} \Gamma_{n}$ is an Ore domain. Therefore it is possible to define the right ring of fractions of $\mathbb{Z} \Gamma_{n}$ (see [Co, Cor. 1.3.3]), which we will denote by $\mathcal{K}_{n}$. The short exact sequence $0 \rightarrow \mathbb{Z} \Gamma_{n} \rightarrow \mathcal{K}_{n} \rightarrow \mathcal{K}_{n} / \mathbb{Z} \Gamma_{n} \rightarrow 0$ gives rise to the Bockstein sequence

$$
\begin{aligned}
H_{2}\left(E(K) ; \mathcal{K}_{n}\right) & \longrightarrow H_{2}\left(E(K) ; \mathcal{K}_{n} / \mathbb{Z} \Gamma_{n}\right) \\
& \stackrel{B}{\longrightarrow} H_{1}\left(E(K) ; \mathbb{Z} \Gamma_{n}\right) \longrightarrow H_{1}\left(E(K) ; \mathcal{K}_{n}\right) .
\end{aligned}
$$

Since the higher-order Alexander modules of a knot are torsion modules, it follows that $H_{i}\left(E(K) ; \mathcal{K}_{n}\right)=0$, for $i=1,2$ ([C], Cor. 3.12). Therefore the Bockstein map, $B: H_{2}\left(E(K) ; \mathcal{K}_{n} / \mathbb{Z} \Gamma_{n}\right) \rightarrow H_{1}\left(E(K) ; \mathbb{Z} \Gamma_{n}\right)$, is an isomorphism.

Let $\mathcal{A}_{n}(K)^{\#} \equiv \overline{\operatorname{Hom}_{\mathbb{Z} \Gamma_{n}}\left(\mathcal{A}_{n}(K), \mathcal{K}_{n} / \mathbb{Z} \Gamma_{n}\right)}$, where given any left $\mathcal{R}$-module $\mathcal{M}, \overline{\mathcal{M}}$ represents the usual associated right $\mathcal{R}$-module resulting from the involution 
of $\mathcal{R}$. We now give the precise definition of the symmetric linking forms defined on the higher-order Alexander modules.

Definition 2.2. The $n$th higher-order linking form, $\mathscr{B}_{n}: \mathcal{A}_{n}(K) \rightarrow \mathcal{A}_{n}(K)^{\#}$, for a knot $K$, is the composition of the following maps:

$$
\begin{aligned}
\mathcal{A}_{n}(K) \stackrel{B^{-1}}{\longrightarrow} & H_{2}\left(E(K) ; \mathcal{K}_{n} / \mathbb{Z} \Gamma_{n}\right) \stackrel{\pi}{\longrightarrow} H_{2}\left(E(K), \partial E(K) ; \mathcal{K}_{n} / \mathbb{Z} \Gamma_{n}\right) \\
\stackrel{P . D .}{\longrightarrow} & H^{1}\left(E(K) ; \mathcal{K}_{n} / \mathbb{Z} \Gamma_{n}\right) \stackrel{\kappa}{\longrightarrow} \mathcal{A}_{n}(K)^{\#},
\end{aligned}
$$

where P.D. is the Poincare duality isomorphism and $\kappa$ is the Kronecker evaluation map. We will often denote $\left[\mathcal{B} \ell_{n}(x)\right](y)$ by $\mathcal{B} \ell_{n}(x, y)$.

The higher-order linking forms that we consider differ from those defined by T. Cochran in [C] and T. Cochran, K. Orr, and P. Teichner in [COT1] because we do not localize the coefficients and because the higher-order linking forms that we consider are canonically associated to $K$, unlike those that were the focus of [COT1]. Furthermore, since $\mathbb{Z} \Gamma_{n}$ is not a PID, our linking forms may be singular.

\section{Genetic infection}

In order to construct the desired examples, we use a satellite technique, that was called genetic infection in [C]. Let $K$ and $J$ be fixed knots, and let $\eta$ be an embedded oriented circle in $S^{3} \backslash K$ which is itself unknotted in $S^{3}$. Since $\eta$ is unknotted in $S^{3}$, it bounds a disc, $D$, in $S^{3}$, which we can choose to intersect $K$ transversely. We construct a new knot by tying the strands of $K$ that pierce $D$ into the knot $J$. That is, we replace the strands of $K$ that intersect a small neighborhood of $D$ with untwisted parallels of a knotted arc with oriented knot type $J$. We call the resulting knot the result of infecting $K$ by $J$ along $\eta$, denoted by $K(\eta, J)$. Alternatively, we can view this construction from a surgery point of view. Beginning with the exterior of $K$, $E(K)$, delete the interior of a tubular neighborhood of $\eta$, and replace it with the exterior of $J, E(J)$, identifying the meridian of $J$ with the longitude of $\eta$, and the longitude of $J$ with the inverse of the meridian of $\eta$. The result is the exterior of $K(\eta, J)$. This surgery description is better suited for our purposes.

Since there is a degree one map (rel boundary) $E(J) \rightarrow E$ (unknot), there is a degree one map $f: E(K(\eta, J)) \rightarrow E(K)$, which is the identity outside of $E(J)$.

Proposition 3.1 ([C], Thm. 8.1). If $\eta \in \pi_{1}(E(K))^{(n)}$, then the map $f$ induces an isomorphism:

$$
f_{*}: \frac{\pi_{1}(E(K(\eta, J)))}{\pi_{1}(E(K(\eta, J)))^{(n+1)}} \longrightarrow \frac{\pi_{1}(E(K))}{\pi_{1}(E(K))^{(n+1)}} .
$$


Therefore we will use $\Gamma_{n}$ to denote both groups. The following composition of maps defines coefficient systems on $E(J), E(K(\eta, J))$, and $E(K)$ :

$$
\pi_{1}(E(J)) \stackrel{i_{*}}{\longrightarrow} \pi_{1}(E(K(\eta, J))) \stackrel{f_{*}}{\longrightarrow} \pi_{1}(E(K)) \stackrel{\phi}{\longrightarrow} \Gamma_{n} .
$$

The following results demonstrate the relationship between genetic infection and the higher-order Alexander modules. We include the proofs from $[\mathrm{C}]$ because some of our results will be proved using similar techniques.

Corollary 3.2. If $\eta \in \pi_{1}(E(K))^{(n)}$, then $f: E(K(\eta, J)) \rightarrow E(K)$ induces isomorphisms between the $i$-th order Alexander modules of $K(\eta, J)$ and $K$, for $0 \leq i \leq n-1$.

Proof. Let $G=\pi_{1}(E(K))$ and $\widehat{G}=\pi_{1}(E(K(\eta, J)))$. We have the following commutative diagram with exact rows.

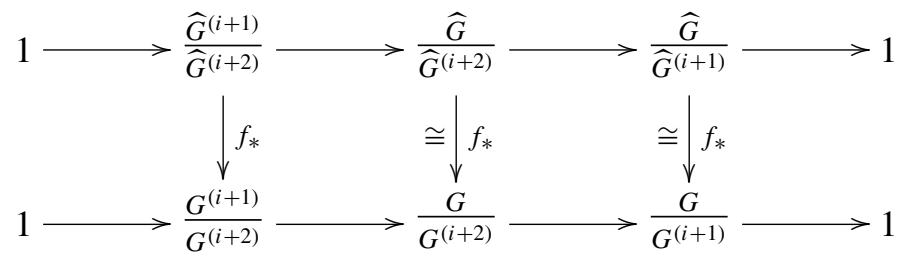

For $0 \leq i \leq n-1$, the middle and right vertical maps are isomorphisms by Proposition 3.1. Therefore $f_{*}: \frac{\widehat{G}^{(i+1)}}{\widehat{G}^{(i+2)}} \longrightarrow \frac{G^{(i+1)}}{G^{(i+2)}}$ is an isomorphism. That is, $\mathcal{A}_{i}(K(\eta, J)) \cong \mathcal{A}_{i}(K)$.

In the proof of the next theorem we will require the following lemma, which we state without proof.

Lemma 3.3 ([C], Lemma 8.3). If $\eta \in \pi_{1}(E(K))^{(n)}$ and $\eta \notin \pi_{1}(E(K))^{(n+1)}$, then the inclusion $\partial E(J) \rightarrow E(J)$ induces an isomorphism on $H_{0}\left(-; \mathbb{Z} \Gamma_{n}\right)$ and the trivial map on $H_{1}\left(-; \mathbb{Z} \Gamma_{n}\right)$.

Theorem 3.4 ([C], Thm. 8.2). If $\eta \in \pi_{1}(E(K))^{(n)}$ and $\eta \notin \pi_{1}(E(K))^{(n+1)}$, then

$$
H_{1}\left(E(K(\eta, J)) ; \mathbb{Z} \Gamma_{n}\right) \cong \mathcal{A}_{n}(K) \oplus H_{1}\left(E(J) ; \mathbb{Z} \Gamma_{n}\right) .
$$

Proof. Let $E(\eta)$ denote the result of deleting the interior of a tubular neighborhood of $\eta$ from the exterior of $K$. Using the surgery description of genetic infection, we have $E(K(\eta, J)) \cong E(J) \cup_{\partial E(J)} E(\eta)$. Since infecting with the unknot leaves the knot unchanged, we can view the exterior of $K$ as the union of the exterior of the unknot, $U$, and $E(\eta)$. That is, $E(K(\eta, J)) \cong E(U) \cup_{\partial E(J)} E(\eta)$. Of course, $E(U)$ is just a solid torus. 
We consider the Mayer-Vietoris sequence with $\mathbb{Z} \Gamma_{n}$ coefficients for $E(K(\eta, J)) \cong$ $E(J) \cup_{\partial E(J)} E(\eta):$

$$
H_{1}(E(K(\eta, J))) \stackrel{\partial_{*}}{\longrightarrow} H_{0}(\partial E(J)) \stackrel{\left(\psi_{1}, \psi_{2}\right)}{\longrightarrow} H_{0}(E(J)) \oplus H_{0}(E(\eta)) .
$$

By Lemma 3.3, $\psi_{1}: H_{0}(\partial E(J)) \rightarrow H_{0}(E(J))$ is an isomorphism. Therefore

$$
\partial_{*}: H_{1}(E(K(\eta, J))) \longrightarrow H_{0}(\partial E(J))
$$

is the trivial map. Similarly, since $E(K) \cong E(U) \cup_{\partial E(J)} E(\eta)$,

$$
\partial_{*}: H_{1}(E(K)) \longrightarrow H_{0}(\partial E(J))
$$

is the trivial map. Thus we have the following diagram.

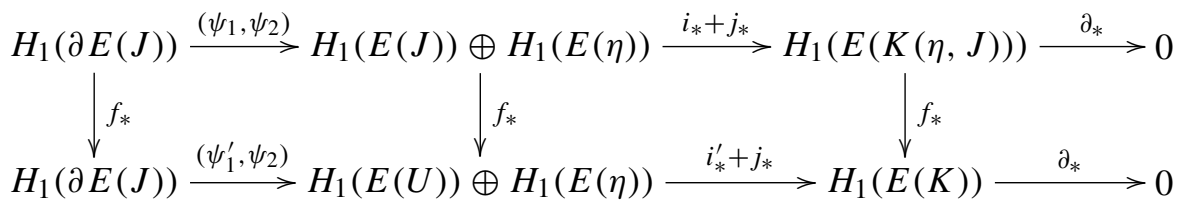

Lemma 3.3 states that $\psi_{1}: H_{1}(\partial E(J)) \rightarrow H_{1}(E(J))$ is the trivial map. Therefore $\operatorname{im}\left(\psi_{1}, \psi_{2}\right)=0 \oplus \psi_{2}\left(H_{1}(\partial E(J))\right)$. Hence $H_{1}(E(K(\eta, J))) \cong H_{1}(E(J)) \oplus$ $\frac{H_{1}(E(\eta))}{\psi_{2}\left(H_{1}(\partial E(J))\right)}$. Similarly, $H_{1}(E(K)) \cong H_{1}(E(U)) \oplus \frac{H_{1}(E(\eta))}{\psi_{2}\left(H_{1}(\partial E(J))\right)}$. Notice that since $f$ is the identity on $\partial E(J)$ and $E(\eta), f_{*} \circ \psi_{2}=\psi_{2} \circ f_{*}$. However, since $\eta \notin \pi_{1}(E(K))^{(n+1)}, \mu_{J}$, which is the generator of $\pi_{1}(E(U) ; \mathbb{Z})$, gets unwound in the $\Gamma_{n}$-cover. Hence $H_{1}(E(U))=0$. Therefore $H_{1}\left(E(K(\eta, J)) ; \mathbb{Z} \Gamma_{n}\right) \cong$ $H_{1}\left(E(J) ; \mathbb{Z} \Gamma_{n}\right) \oplus H_{1}\left(E(K) ; \mathbb{Z} \Gamma_{n}\right)$.

Corollary 3.5. If $\eta \in \pi_{1}(E(K))^{(n)}$ and $\eta \notin \pi_{1}(E(K))^{(n+1)}$, then

$$
0 \rightarrow H_{1}\left(E(J) ; \mathbb{Z} \Gamma_{n}\right) \stackrel{i_{*}}{\longrightarrow} H_{1}\left(E(K(\eta, J)) ; \mathbb{Z} \Gamma_{n}\right) \stackrel{f_{*}}{\longrightarrow} H_{1}\left(E(K) ; \mathbb{Z} \Gamma_{n}\right) \rightarrow 0
$$

is a split short exact sequence.

Since $\pi_{1}(E(J))$ is normally generated by the meridian of $J$, it follows that if $\eta \in$ $\pi_{1}(E(K))^{(n)}$, then the image of $\pi_{1}(E(J))$ is contained in $\pi_{1}(E(K))^{(n)}$. Therefore, $\pi_{1}(E(J))^{\prime}$ is in $\pi_{1}(E(K))^{(n+1)}$, and thus in the kernel of the composition:

$$
\pi_{1}(E(J)) \stackrel{i_{*}}{\longrightarrow} \pi_{1}(E(K(\eta, J))) \stackrel{f_{*}}{\longrightarrow} \pi_{1}(E(K)) \stackrel{\phi}{\longrightarrow} \Gamma_{n} .
$$

Hence, we have a ring homomorphism

$$
\mathbb{Z}\left[\frac{\pi_{1}(E(J))}{\pi_{1}(E(J))^{\prime}}\right] \cong \mathbb{Z}\left[t, t^{-1}\right] \stackrel{\psi}{\longrightarrow} \mathbb{Z} \Gamma_{n} .
$$

If $\eta \notin \pi_{1}(E(K))^{(n+1)}$, this is a monomorphism. 
Corollary 3.6. If $\eta \in \pi_{1}(E(K))^{(n)}$, then

$$
\mathcal{A}_{n}(K(\eta, J)) \cong \mathcal{A}_{n}(K) \oplus\left(\mathcal{A}_{0}(J) \otimes_{\mathbb{Z}\left[t, t^{-1}\right]} \mathbb{Z} \Gamma_{n}\right)
$$

where $\mathbb{Z} \Gamma_{n}$ is a left $\mathbb{Z}\left[t, t^{-1}\right]$-module by the homomorphism sending $t$ to $\phi(\eta)$.

Proof. If $\eta \in \pi_{1}(E(K))^{(n+1)}$, then $\mathcal{A}_{n}(K(\eta, J)) \cong \mathcal{A}_{n}(K)$ by Corollary 3.2. Also in this case, $\phi(\eta)=1$. Therefore, $\mathcal{A}_{0}(J) \otimes_{\mathbb{Z}\left[t, t^{-1}\right]} \mathbb{Z} \Gamma_{n}=0$, since $\mathcal{A}_{0}(J)$ is presented by $V-t V^{t}$ and $\operatorname{det}\left(V-V^{t}\right)=1$, where $V$ is a Seifert matrix for $J$.

If $\eta \notin \pi_{1}(E(K))^{(n+1)}$, then $H_{1}\left(E(K(\eta, J)) ; \mathbb{Z} \Gamma_{n}\right) \cong \mathcal{A}_{n}(K) \oplus H_{1}\left(E(J) ; \mathbb{Z} \Gamma_{n}\right)$ by Theorem 3.4. Furthermore, if $\widetilde{E(J)}$ is the universal cover of $E(J)$,

$$
\begin{aligned}
H_{1}\left(E(J) ; \mathbb{Z} \Gamma_{n}\right) & =H_{1}\left(C_{*}(\widetilde{E(J)}) \otimes_{\mathbb{Z} \pi_{1}(E(J))} \mathbb{Z} \Gamma_{n}\right) \\
& \cong H_{1}\left(C_{*}(\widetilde{E(J)}) \otimes_{\mathbb{Z} \pi_{1}(E(J))} \mathbb{Z}\left[t, t^{-1}\right] \otimes_{\mathbb{Z}\left[t, t^{-1}\right]} \mathbb{Z} \Gamma_{n}\right)
\end{aligned}
$$

(cf. [HS, p. 109]).

Since $\eta \notin \pi_{1}(E(K))^{n+1}$, it follows that $\mathbb{Z}\left[t, t^{-1}\right] \stackrel{\psi}{\rightarrow} \mathbb{Z} \Gamma_{n}$ is a monomorphism. It follows from [P, Lemma 1.3] that $\mathbb{Z} \Gamma_{n}$ is a free, and therefore flat $\mathbb{Z}\left[t, t^{-1}\right]$-module. Hence,

$$
\begin{aligned}
H_{1}\left(E(J) ; \mathbb{Z} \Gamma_{n}\right) & \cong H_{1}\left(C_{*}(\widetilde{E(J)}) \otimes_{\mathbb{Z} \pi_{1}(E(J))} \mathbb{Z}\left[t, t^{-1}\right]\right) \otimes_{\mathbb{Z}\left[t, t^{-1}\right]} \mathbb{Z} \Gamma_{n} \\
& \cong H_{1}\left(E(J) ; \mathbb{Z}\left[t, t^{-1}\right]\right) \otimes_{\mathbb{Z}\left[t, t^{-1}\right]} \mathbb{Z} \Gamma_{n} \\
& \cong \mathcal{A}_{0}(J) \otimes_{\mathbb{Z}\left[t, t^{-1}\right]} \mathbb{Z} \Gamma_{n} .
\end{aligned}
$$

\section{The effect of genetic infection on the higher-order linking forms}

The idea behind the construction of our examples is to infect the same knot $K$ along the same element $\eta \in \pi_{1}(E(K))^{(n)}, \eta \notin \pi_{1}(E(K))^{(n+1)}$ by two different knots $J_{1}$, $J_{2}$ that have isomorphic classical Alexander modules. Corollary 3.6 implies that the results of these infections will have isomorphic $i$-th order Alexander modules for $0 \leq i \leq n$. We need to choose the knots so that the higher-order linking forms of the results of the infections are not isomorphic. In this section, we will determine the effect of genetic infection on the higher-order linking forms in order to determine what the desired conditions are on the infecting knots.

Theorem 4.1. If $\eta \in \pi_{1}(E(K))^{(n)}$, then $f: E(K(\eta, J)) \rightarrow E(K)$ induces isomorphisms between the $i$-th order linking forms of $K(\eta, J)$ and $K$, for $0 \leq i \leq n-1$. 
Proof. We have the following diagram.

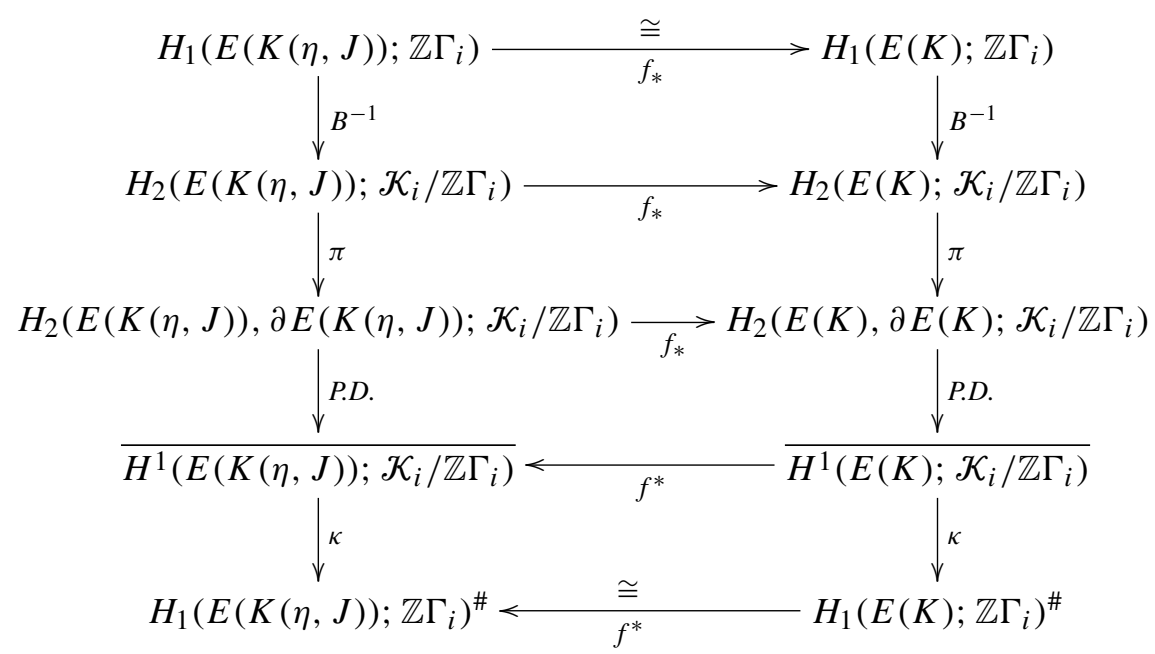

By the naturality of the Bockstein isomorphism, $\pi$, the Poincaré duality isomorphism, and the Kronecker map, $\mathscr{B} \ell_{i}(K(\eta, J))=f^{*} \circ \mathscr{B} \ell_{i}(K) \circ f_{*}$. Since, by Corollary 3.2, $f$ induces isomorphisms between the $i$-th order Alexander modules of $K(\eta, J)$ and $K$, for $0 \leq i<n$, it follows that $f$ induces isomorphisms between $\mathscr{B} \ell_{i}(K(\eta, J))$ and $\mathcal{B} \ell_{i}(K)$.

From now on, we will regard $n$ as fixed and restrict our attention to the case where $\eta \in \pi_{1}(E(K))^{(n)}$ and $\eta \notin \pi_{1}(E(K))^{(n+1)}$. As a result, we will suppress $n$ from our notation for the higher-order linking forms. Therefore, let

$$
\mathcal{B} \ell_{K(\eta, J)}: H_{1}\left(E(K(\eta, J)) ; \mathbb{Z} \Gamma_{n}\right) \longrightarrow H_{1}\left(E(K(\eta, J)) ; \mathbb{Z} \Gamma_{n}\right)^{\#}
$$

and

$$
\mathcal{B} \ell_{K}: H_{1}\left(E(K) ; \mathbb{Z} \Gamma_{n}\right) \longrightarrow H_{1}\left(E(K) ; \mathbb{Z} \Gamma_{n}\right)^{\#}
$$

denote the higher-order linking forms for $K(\eta, J)$ and $K$, respectively.

Recall that the following composition of maps defines a coefficient system on $E(J)$ :

$$
\pi_{1}(E(J)) \stackrel{i_{*}}{\longrightarrow} \pi_{1}(E(K(\eta, J))) \stackrel{f_{*}}{\longrightarrow} \pi_{1}(E(K)) \stackrel{\phi}{\longrightarrow} \Gamma_{n} .
$$

This coefficient system is non-trivial if $\eta \notin \pi_{1}(E(K))^{(n+1)}$.

Proposition 4.2. If $\eta \in \pi_{1}(E(K))^{(n)}$ and $\eta \notin \pi_{1}(E(K))^{(n+1)}$, then $H_{1}\left(E(J) ; \mathbb{Z} \Gamma_{n}\right)$ is a $\mathbb{Z} \Gamma_{n}$-torsion module. 
Proof. Since $\eta \in \pi_{1}(E(K))^{(n)}$ and $\eta \notin \pi_{1}(E(K))^{(n+1)}$ we have a ring monomorphism $\mathbb{Z}\left[t, t^{-1}\right] \rightarrow \mathbb{Z} \Gamma_{n}$. Therefore we have $\mathbb{Z}\left[t, t^{-1}\right]$-module homomorphisms $\mathbb{Q}(t) \hookrightarrow \mathcal{K}_{n}$. Furthermore, from the proof of Corollary 3.6, $H_{1}\left(E(J) ; \mathbb{Z} \Gamma_{n}\right) \cong$ $\mathcal{A}_{0}(J) \otimes_{\mathbb{Z}\left[t, t^{-1}\right]} \mathbb{Z} \Gamma_{n}$ where $\mathbb{Z} \Gamma_{n}$ is a left $\mathbb{Z}\left[t, t^{-1}\right]$-module by the homomorphism sending $t$ to $\phi(\eta)$. Hence, we have the following:

$$
\begin{aligned}
& \mathcal{A}_{0}(J) \otimes_{\mathbb{Z}\left[t, t^{-1}\right]} \mathbb{Z} \Gamma_{n} \otimes_{\mathbb{Z} \Gamma_{n}} \mathcal{K}_{n} \cong \mathcal{A}_{0}(J) \otimes_{\mathbb{Z}\left[t, t^{-1}\right]} \mathcal{K}_{n} \\
& \\
& \cong \mathcal{A}_{0}(J) \otimes_{\mathbb{Z}\left[t, t^{-1}\right]} \mathbb{Q}(t) \otimes_{\mathbb{Q}(t)} \mathcal{K}_{n} \\
& \cong 0
\end{aligned}
$$

since $\mathcal{A}_{0}(J)$ is a $\mathbb{Z}\left[t, t^{-1}\right]$-torsion module.

Again, the short exact sequence $0 \rightarrow \mathbb{Z} \Gamma_{n} \rightarrow \mathcal{K}_{n} \rightarrow \mathcal{K}_{n} / \mathbb{Z} \Gamma_{n} \rightarrow 0$ gives rise to a Bockstein sequence.

$H_{2}\left(E(J) ; \mathcal{K}_{n}\right) \longrightarrow H_{2}\left(E(J) ; \mathcal{K}_{n} / \mathbb{Z} \Gamma_{n}\right) \stackrel{B}{\longrightarrow} H_{1}\left(E(J) ; \mathbb{Z} \Gamma_{n}\right) \longrightarrow H_{1}\left(E(J) ; \mathcal{K}_{n}\right)$

Corollary 4.3. If $\eta \in \pi_{1}(E(K))^{(n)}$ and $\eta \notin \pi_{1}(E(K))^{(n+1)}$, then for $i=1,2$,

$$
H_{i}\left(E(J) ; \mathcal{K}_{n}\right)=0 .
$$

Proof. Since $\mathcal{K}_{n}$ is a flat $\mathbb{Z} \Gamma_{n}$-module (see [Ste, Prop. II.3.5]), $H_{1}\left(E(K) ; \mathcal{K}_{n}\right) \cong$ $H_{1}\left(E(K) ; \mathbb{Z} \Gamma_{n}\right) \otimes_{\mathbb{Z} \Gamma_{n}} \mathcal{K}_{n}=0$ since $\mathcal{A}_{n}(K)$ is a torsion module. Similarly, it follows from Prop. 3.7 of [C] that $H_{0}\left(\partial E(K) ; \mathcal{K}_{n}\right)=0$. Therefore by the long exact sequence of a pair, $H_{1}\left(E(K), \partial E(K) ; \mathcal{K}_{n}\right)=0$. By Poincaré duality and the Universal Coefficient Theorem for modules over the (noncommutative) principal ideal domain $\mathcal{K}_{n}$ [DK, pp. 44,102], we have

$$
H_{2}\left(E(K) ; \mathcal{K}_{n}\right) \cong \operatorname{Hom}_{\mathcal{K}_{n}}\left(H_{1}\left(E(K), \partial E(K) ; \mathcal{K}_{n}\right), \mathcal{K}_{n}\right)=0 .
$$

Corollary 4.4. If $\eta \in \pi_{1}(E(K))^{(n)}$ and $\eta \notin \pi_{1}(E(K))^{(n+1)}$, then the Bockstein map, $B: H_{2}\left(E(J) ; \mathcal{K}_{n} / \mathbb{Z} \Gamma_{n}\right) \rightarrow H_{1}\left(E(J) ; \mathbb{Z} \Gamma_{n}\right)$, is an isomorphism.

Definition 4.5. If $\eta \in \pi_{1}(E(K))^{(n)}$ and $\eta \notin \pi_{1}(E(K))^{(n+1)}$, define $\mathcal{B} \ell_{K(\eta, J)}^{\otimes}$ : $H_{1}\left(E(J) ; \mathbb{Z} \Gamma_{n}\right) \rightarrow H_{1}\left(E(J) ; \mathbb{Z} \Gamma_{n}\right)^{\#}$, to be the composition of the following maps:

$$
\begin{aligned}
H_{1}\left(E(J) ; \mathbb{Z} \Gamma_{n}\right) \stackrel{B^{-1}}{\longrightarrow} & H_{2}\left(E(J) ; \mathcal{K}_{n} / \mathbb{Z} \Gamma_{n}\right) \stackrel{\pi}{\longrightarrow} H_{2}\left(E(J), \partial E(J) ; \mathcal{K}_{n} / \mathbb{Z} \Gamma_{n}\right) \\
\stackrel{\text { P.D. }}{\longrightarrow} & \frac{\kappa}{H^{1}\left(E(J) ; \mathcal{K}_{n} / \mathbb{Z} \Gamma_{n}\right)} \stackrel{\kappa}{\longrightarrow} H_{1}\left(E(J) ; \mathbb{Z} \Gamma_{n}\right)^{\#},
\end{aligned}
$$

where P.D. is the Poincaré duality isomorphism and $\kappa$ is the Kronecker evaluation map. We remark that the coefficient system that we are using is defined using $K(\eta, J)$, and therefore $\mathcal{B} \ell_{K(\eta, J)}^{\otimes}$ does indeed depend on $K$ and $\eta$, as well as $J$. 
Let $g$ be a splitting for the exact sequence in Corollary 3.5. That is, $f_{*} \circ g=\mathrm{id}$.

Theorem 4.6. If $\eta \in \pi_{1}(E(K))^{(n)}$ and $\eta \notin \pi_{1}(E(K))^{(n+1)}$, then $B \ell_{K(\eta, J)} \cong$ $\mathcal{B} \ell_{K(\eta, J)}^{\otimes} \oplus \mathscr{B} \ell_{K}$. That is,

$$
\mathscr{B} \ell_{K(\eta, J)}^{\otimes}\left(x_{1}, y_{1}\right)+\mathscr{B} \ell_{K}\left(x_{2}, y_{2}\right)=\mathscr{B} \ell_{K(\eta, J)}\left(i_{*}\left(x_{1}\right)+g\left(x_{2}\right), i_{*}\left(y_{1}\right)+g\left(y_{2}\right)\right) .
$$

Proof. We have the following diagram.

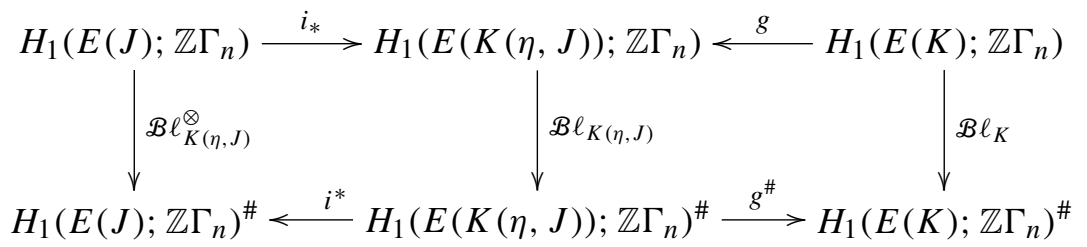

where $g^{\#}$ is the dual of $g$. Notice that since $f_{*} \circ g=\mathrm{id}$, it follows that $g^{\#} \circ f^{*}=$ id. The isomorphism in the theorem will be given by $i_{*} \oplus g: H_{1}\left(E(K) ; \mathbb{Z} \Gamma_{n}\right) \oplus$ $H_{1}\left(E(J) ; \mathbb{Z} \Gamma_{n}\right) \rightarrow H_{1}\left(E(K(\eta, J)) ; \mathbb{Z} \Gamma_{n}\right)$. Hence the theorem will follow from the following four claims.

(1) $g^{\#} \circ \mathscr{B} \ell_{K(\eta, J)} \circ g=\mathscr{B} \ell_{K}$ which establishes $\mathscr{B} \ell_{K(\eta, J)}\left(g\left(x_{2}\right), g\left(y_{2}\right)\right)=$ $\mathscr{B} \ell_{K}\left(x_{2}, y_{2}\right)$;

(2) $i^{*} \circ \mathscr{B} \ell_{K(\eta, J)} \circ i_{*}=\mathscr{B} \ell_{K(\eta, J)}^{\otimes}$ which establishes $\mathscr{B} \ell_{K(\eta, J)}\left(i_{*}\left(x_{1}\right), i_{*}\left(y_{1}\right)\right)=$ $\mathcal{B} \ell_{K(\eta, J)}^{\otimes}\left(x_{1}, y_{1}\right)$;

(3) $g^{\#} \circ \mathscr{B} \ell_{K(\eta, J)} \circ i_{*}=0$ which establishes $\mathscr{B} \ell_{K(\eta, J)}\left(i_{*}\left(x_{1}\right), g\left(y_{2}\right)\right)=0$;

(4) $i^{*} \circ \mathscr{B} \ell_{K(\eta, J)} \circ g=0$ which establishes $\mathscr{B} \ell_{K(\eta, J)}\left(g\left(x_{2}\right), i_{*}\left(y_{1}\right)\right)=0$.

We have the following diagram.

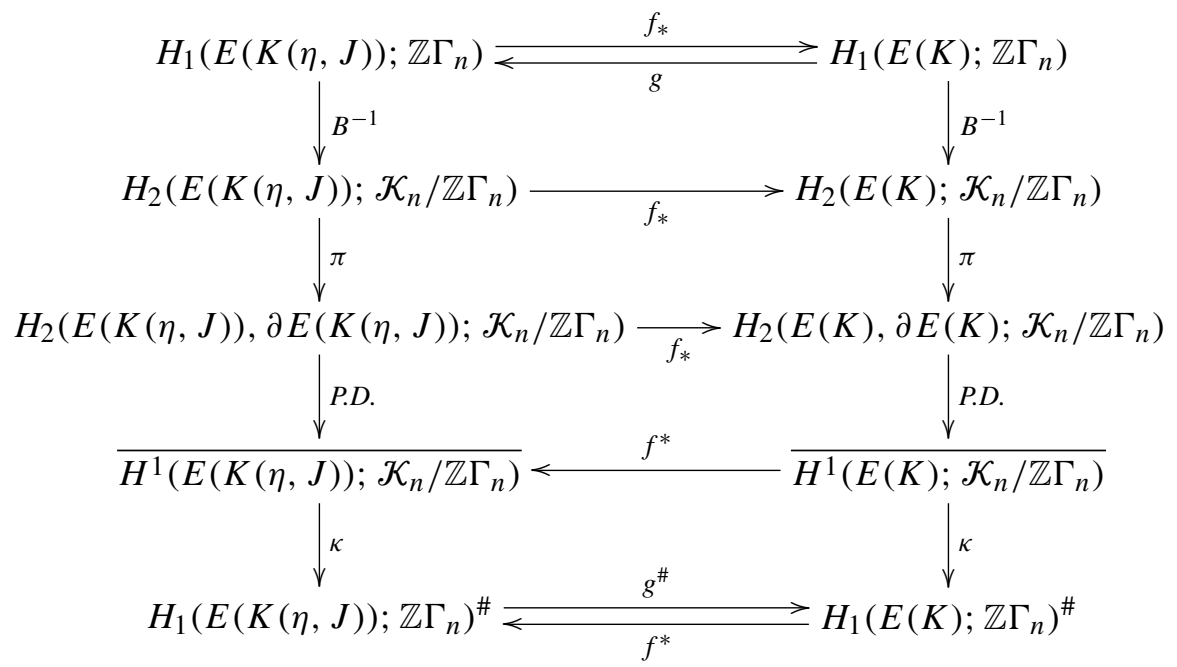


By the naturality of the Bockstein isomorphism, $\pi$, the Poincaré duality isomorphism, and the Kronecker map, $f^{*} \circ \mathscr{B} \ell_{K} \circ f_{*}=\mathscr{B} \ell_{K(\eta, J)}$. So $g^{\#} \circ f^{*} \circ \mathscr{B} \ell_{K} \circ f_{*} \circ g=$ $g^{\#} \circ \mathscr{B} \ell_{K(\eta, J)} \circ g$. Since $f_{*} \circ g=$ id and $g^{\#} \circ f^{*}=$ id, it follows that $g^{\#} \circ \mathscr{B} \ell_{K(\eta, J)} \circ g=$ $\mathscr{B} \ell_{K}$. Hence the first claim is proved.

Consider the following diagram.

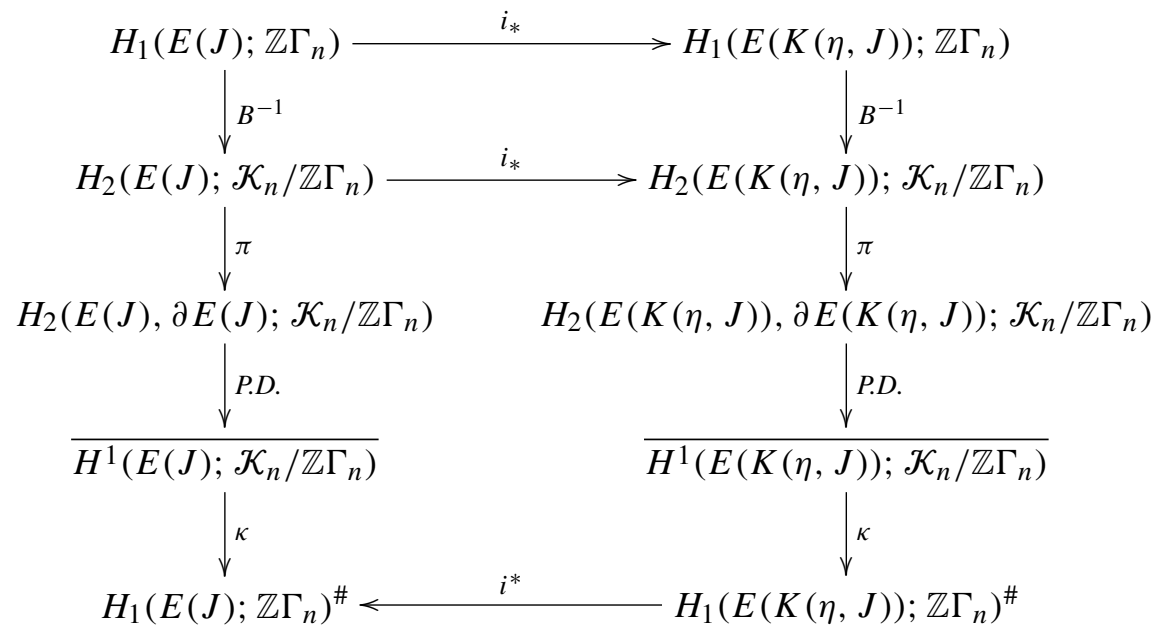

By the naturality of the Bockstein homomorphism, $B^{-1} \circ i_{*}=i_{*} \circ B^{-1}$. Consider the intersection pairing (see, for example, [D]) $I_{E(K(\eta, J))}: H_{2}\left(E(K(\eta, J)) ; \mathcal{K}_{n} / \mathbb{Z} \Gamma_{n}\right) \rightarrow$ $H_{1}\left(E(K(\eta, J)) ; \mathbb{Z} \Gamma_{n}\right)^{\#}$ on $E(K(\eta, J))$ given by $I_{E(K(\eta, J))}=\kappa \circ P . D$. $\circ \pi$. Similarly, we have the intersection form $I_{E(J)}=\kappa \circ P . D . \circ \pi: H_{2}\left(E(J) ; \mathcal{K}_{n} / \mathbb{Z} \Gamma_{n}\right) \rightarrow$ $H_{1}\left(E(J) ; \mathbb{Z} \Gamma_{n}\right)^{\#}$ on $E(J)$. Since $i: E(J) \rightarrow E(K(\eta, J))$ is an embedding, $I_{E(J)}(x, y)=I_{E(K(\eta, J))}\left(i_{*}(x), i_{*}(y)\right)$. Therefore, $\kappa \circ P . D . \circ \pi=i^{*} \circ(\kappa \circ P . D . \circ \pi) \circ i_{*}$. Thus,

$$
\begin{aligned}
\mathcal{B}_{K(\eta, J)}^{\otimes} & =(\kappa \circ P . D . \circ \pi) \circ B^{-1} \\
& =i^{*} \circ(\kappa \circ P . D . \circ \pi) \circ i_{*} \circ B^{-1} \\
& =i^{*} \circ(\kappa \circ P . D . \circ \pi) \circ B^{-1} \circ i_{*} \\
& =i^{*} \circ B \ell_{K(\eta, J)} \circ i_{*} .
\end{aligned}
$$

Therefore the second claim is proved.

Finally consider the following diagram.

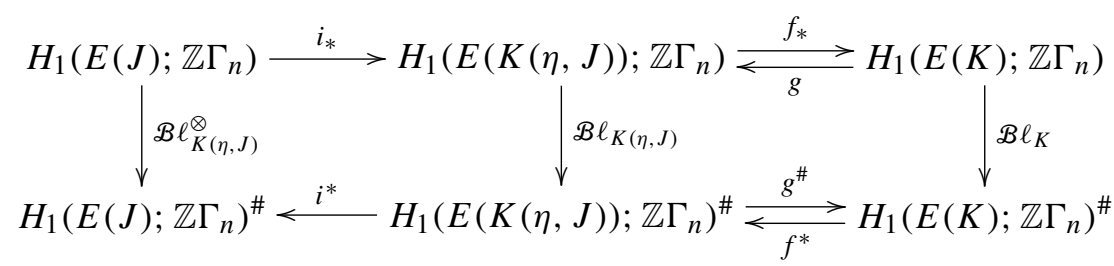


Since $f^{*} \circ \mathscr{B} \ell_{K} \circ f_{*}=\mathscr{B} \ell_{K(\eta, J)}$, it follows that $g^{\#} \circ \mathscr{B} \ell_{K(\eta, J)} \circ i_{*}=g^{\#} \circ f^{*} \circ$ $\mathscr{B} \ell_{K} \circ f_{*} \circ i_{*}$. But by Corollary $3.5, f_{*} \circ i_{*}=0$. Therefore $g^{\#} \circ \mathscr{B} \ell_{K(\eta, J)} \circ i_{*}=0$. And since $f_{*} \circ i_{*}=0$, we also have that $i^{*} \circ f^{*}=0$. Therefore, $i^{*} \circ \mathscr{B} \ell_{K(\eta, J)} \circ g=$ $i^{*} \circ f^{*} \circ \mathscr{B}_{K} \circ f_{*} \circ g=0$.

Recall that if $\eta \in \pi_{1}(E(K))^{(n)}$ and $\eta \notin \pi_{1}(E(K))^{(n+1)}$ we have a ring monomorphism $\psi: \mathbb{Z}\left[t, t^{-1}\right] \rightarrow \mathbb{Z} \Gamma_{n}$. Therefore we have $\mathbb{Z}\left[t, t^{-1}\right]$-module homomorphisms:

$$
\begin{gathered}
\mathbb{Q}(t) \longleftrightarrow \mathcal{K}_{n}, \quad \mathbb{Q}(t) / \mathbb{Z}\left[t, t^{-1}\right] \stackrel{\bar{\psi}}{\longrightarrow} \mathcal{K}_{n} / \mathbb{Z} \Gamma_{n}, \\
H_{*}\left(E(J) ; \mathbb{Z}\left[t, t^{-1}\right]\right) \stackrel{\psi_{*}}{\longrightarrow} H_{*}\left(E(J) ; \mathbb{Z} \Gamma_{n}\right) .
\end{gathered}
$$

We will state the following theorem without proof since the proof is quite technical and it will not be needed for our main result. It shows that $\mathscr{B} \ell_{K(\eta, J)}^{\otimes}$ is determined by the classical Blanchfield form for $J$.

Theorem 4.7. If $\eta \in \pi_{1}(E(K))^{(n)}$ and $\eta \notin \pi_{1}(E(K))^{(n+1)}$,

$$
\mathcal{B} \ell_{K(\eta, J)}^{\otimes}\left(\psi_{*}\left(x_{1}\right), \psi_{*}\left(x_{2}\right)\right)=\bar{\psi}\left(\mathscr{B} \ell_{J}\left(x_{1}, x_{2}\right)\right),
$$

where $\mathscr{B} \ell_{J}$ is the classical Blanchfield linking form for $J$.

\section{Reducing from $B \ell_{K(\eta, J)}$ to $B \ell_{K(\eta, J)}^{\otimes}$}

Recall that our strategy is to find knots $J_{1}$ and $J_{2}$ such that $\mathcal{A}_{0}\left(J_{1}\right) \cong \mathcal{A}_{0}\left(J_{2}\right)$ but $\mathscr{B} \ell_{K\left(\eta, J_{1}\right)} \nsucceq \mathscr{B} \ell_{K\left(\eta, J_{2}\right)}$. From Theorems 4.6 and 4.7, we know that if the classical Blanchfield linking forms of $J_{1}$ and $J_{2}$ are isomorphic, then $\mathscr{B} \ell_{K\left(\eta, J_{1}\right)}^{\otimes} \cong \mathscr{B} \ell_{K\left(\eta, J_{2}\right)}^{\otimes}$ and, therefore, $\mathscr{B} \ell_{K\left(\eta, J_{1}\right)} \cong \mathscr{B} \ell_{K\left(\eta, J_{2}\right)}$. However, the converses of these implications may not follow. That is, it may not be sufficient to choose $J_{1}$ and $J_{2}$ with nonisomorphic classical Blanchfield linking forms. In this section, we find conditions on $K$ that ensure that $\mathscr{B} \ell_{K\left(\eta, J_{1}\right)} \cong \mathscr{B} \ell_{K\left(\eta, J_{2}\right)}$ if and only if $\mathscr{B} \ell_{K\left(\eta, J_{1}\right)}^{\otimes} \cong \mathscr{B} \ell_{K\left(\eta, J_{2}\right)}^{\otimes}$.

Proposition 5.1. If $\eta \in \pi_{1}(E(K))^{(n)}, \eta \notin \pi_{1}(E(K))^{(n+1)}$, and $n \geq 1$, then $H_{1}\left(E(J) ; \mathbb{Z} \Gamma_{n}\right)$ is a right $\mathbb{Z} \Gamma_{n}^{\prime}$-torsion module, where $\Gamma_{n}^{\prime}=\left[\Gamma_{n}, \Gamma_{n}\right]$. That is, for any $\alpha \in H_{1}\left(E(J) ; \mathbb{Z} \Gamma_{n}\right)$, there is a non-zero $\gamma_{\alpha}^{\prime} \in \mathbb{Z} \Gamma_{n}^{\prime}$ such that $\alpha \gamma_{\alpha}^{\prime}=0$.

Proof. Recall from the proof of Corollary 3.6 that $H_{1}\left(E(J) ; \mathbb{Z} \Gamma_{n}\right) \cong \mathcal{A}_{0}(J) \otimes_{\mathbb{Z}\left[t, t^{-1}\right]}$ $\mathbb{Z} \Gamma_{n}$. Hence, it suffices to consider $\beta \otimes \gamma$ where $\beta \in \mathcal{A}_{0}(J)$ and $\gamma \in \mathbb{Z} \Gamma_{n}$ are nonzero. Let $\Delta_{J}$ be the classical Alexander polynomial of $J$. Since $\eta \in \pi_{1}(E(K))^{(n)} \subset$ $\pi_{1}(E(K))^{\prime}$ and $\eta \notin \pi_{1}(E(K))^{(n+1)}$, it follows that $\psi\left(\Delta_{J}\right) \in \mathbb{Z} \Gamma_{n}^{\prime}$ is not zero. Since 
$\mathbb{Z} \Gamma_{n}^{\prime}$ is a right $\mathbb{Z} \Gamma_{n}-\{0\}$ Ore set [Co, p. 16], there exist $\widehat{\gamma} \in \mathbb{Z} \Gamma_{n}$ and $\gamma^{\prime} \in \mathbb{Z} \Gamma_{n}^{\prime}$ such that $\gamma \cdot \gamma^{\prime}=\psi\left(\Delta_{J}\right) \cdot \widehat{\gamma}$ and $\widehat{\gamma} \neq 0, \gamma \neq 0$. Thus

$$
(\beta \otimes \gamma) \cdot \gamma^{\prime}=\beta \otimes\left(\gamma \cdot \gamma^{\prime}\right)=\beta \otimes\left(\psi\left(\Delta_{J}\right) \cdot \widehat{\gamma}\right)=\left(\beta \cdot \Delta_{J}\right) \otimes \widehat{\gamma}
$$

since $\mathbb{Z} \Gamma_{n}$ is a left $\mathbb{Z}\left[t, t^{-1}\right]$-module via the ring monomorphism $\psi$. However, since $\beta \in \mathcal{A}_{0}(J)$ and $\Delta_{J}$ annihilates the Alexander module, $\beta \cdot \Delta_{J}=0$.

Lemma 5.2. $\mathbb{Z} \Gamma_{n} \cong \mathbb{Z} \pi_{1}(E(K)) \otimes_{\mathbb{Z} \pi_{1}(E(K))^{\prime}} \mathbb{Z} \Gamma_{n}^{\prime}$ as right $\mathbb{Z} \Gamma_{n}^{\prime}$-modules.

Proof. Define $\varphi: \mathbb{Z} \Gamma_{n} \rightarrow \mathbb{Z} \pi_{1}(E(K)) \otimes_{\mathbb{Z} \pi_{1}(E(K))^{\prime}} \mathbb{Z} \Gamma_{n}^{\prime}$ by

$$
\sum_{i} n_{i}\left[g_{i}\right] \mapsto\left(\sum_{i} n_{i} g_{i}\right) \otimes 1,
$$

where $[g]$ represents the coset of $g \in \pi_{1}(E(K))$ in $\Gamma_{n}=\pi_{1}(E(K)) / \pi_{1}(E(K))^{(n+1)}$. First we must show that this is well-defined. If $h_{i} \in \pi_{1}(E(K))^{(n+1)}$ for all $i$, then

$$
\varphi\left(\sum_{i} n_{i}\left[g_{i} h_{i}\right]\right)=\left(\sum_{i} n_{i} g_{i} h_{i}\right) \otimes 1=\sum_{i} n_{i}\left(g_{i} \otimes h_{i}\right)=\sum_{i} n_{i}\left(g_{i} \otimes 1\right) .
$$

For any $g \in \Gamma_{n}$ and $g^{\prime} \in \Gamma_{n}^{\prime}, g g^{\prime} \otimes 1=g \otimes g^{\prime}$. Hence $\varphi\left(\left[g g^{\prime}\right]\right)=\varphi([g]) g^{\prime}$. It is easy to see that $\varphi$ preserves addition. Finally, we define an inverse $\psi$ by

$$
\sum_{i} n_{i} g_{i} \otimes \sum_{j} m_{j} g_{j}^{\prime} \longmapsto \sum_{i, j} n_{i} m_{j}\left[g_{i} g_{j}^{\prime}\right]
$$

Note that $\psi \circ \varphi=$ id and $\varphi \circ \psi=$ id, since

$$
\sum_{i} n_{i} g_{i} \otimes \sum_{j} m_{j} g_{j}^{\prime}=\sum_{i, j} n_{i} m_{j} g_{i} g_{j}^{\prime} \otimes 1 .
$$

Therefore $\varphi$ is a right $\mathbb{Z} \Gamma_{n}^{\prime}$-module isomorphism.

Proposition 5.3 ([C], Prop. 9.3). If $K$ is a fibered knot, then $\mathcal{A}_{n}(K)$ has no $\mathbb{Z} \Gamma_{n}^{\prime}$ torsion. That is, for any $\beta \in \mathcal{A}_{n}(K)$ and $\gamma^{\prime} \in \mathbb{Z} \Gamma_{n}^{\prime}$, if $\beta \gamma^{\prime}=0$, then $\beta=0$ or $\gamma^{\prime}=0$.

Proof. Let $E(K)_{\infty}$ be the infinite cyclic cover, and $\widetilde{E(K)}$ be the universal cover of $E(K)$. Since $K$ is a fibered knot, $E(K)_{\infty}$ is homotopy equivalent to a wedge of circles, $X$. Since $X$ is a 1-complex, $H_{1}\left(X ; \mathbb{Z} \Gamma_{n}^{\prime}\right) \subset C_{1}\left(X ; \mathbb{Z} \Gamma_{n}^{\prime}\right)$ which is a free right $\mathbb{Z} \Gamma_{n}^{\prime}$-module. Therefore $H_{1}\left(E(K)_{\infty} ; \mathbb{Z} \Gamma_{n}^{\prime}\right)$ has no $\mathbb{Z} \Gamma_{n}^{\prime}$-torsion. Furthermore, the following are isomorphic right $\mathbb{Z} \Gamma_{n}^{\prime}$-modules: 


$$
\begin{aligned}
H_{1}\left(E(K)_{\infty} ; \mathbb{Z} \Gamma_{n}^{\prime}\right) & =H_{1}\left(C_{*}(\widetilde{E(K)}) \otimes_{\mathbb{Z} \pi_{1}(E(K))^{\prime}} \mathbb{Z} \Gamma_{n}^{\prime}\right) \\
& \cong H_{1}\left(C_{*}(\widetilde{E(K)}) \otimes_{\mathbb{Z} \pi_{1}(E(K))} \mathbb{Z} \pi_{1}(E(K)) \otimes_{\mathbb{Z} \pi_{1}(E(K))^{\prime}} \mathbb{Z} \Gamma_{n}^{\prime}\right) \\
& \cong H_{1}\left(C_{*}(\widetilde{E(K)}) \otimes_{\mathbb{Z} \pi_{1}(E(K))} \mathbb{Z} \Gamma_{n}\right) \text { by Lemma } 5.2 \\
& =H_{1}\left(E(K) ; \mathbb{Z} \Gamma_{n}\right) .
\end{aligned}
$$

Therefore $\mathcal{A}_{n}(K)$ has no $\mathbb{Z} \Gamma_{n}^{\prime}$-torsion.

Theorem 5.4. Let $K$ be a fibered knot. Suppose $\eta \in \pi_{1}(E(K))^{(n)}, \eta \notin \pi_{1}(E(K))^{(n+1)}$, and $n \geq 1$. If $\mathscr{B} \ell_{K\left(\eta, J_{1}\right)}$ and $\mathscr{B} \ell_{K\left(\eta, J_{2}\right)}$ are isomorphic, then $\mathcal{B} \ell_{K\left(\eta, J_{1}\right)}^{\otimes}$ and $\mathscr{B} \ell_{K\left(\eta, J_{2}\right)}^{\otimes}$ are isomorphic.

Remark 5.5. Before proving the theorem, we remark that for any fibered knot $K$, that is not the unknot, and any $n \geq 1$, there exists an $\eta$ such that $\eta \in \pi_{1}(E(K))^{(n)}$ and $\eta \notin \pi_{1}(E(K))^{(n+1)}$. This is because $K$ being fibered implies that $\pi_{1}(E(K))^{(1)}$ is isomorphic to a free group $F_{k}$ on $k$ generators $(k>1$, since $K$ is not the unknot). Therefore,

$$
\frac{\pi_{1}(E(K))^{(n)}}{\pi_{1}(E(K))^{(n+1)}} \cong \frac{F_{k}^{(n-1)}}{F_{k}^{(n)}},
$$

which is well-known to be non-trivial.

Proof of Theorem 5.4. Suppose $\mathscr{B} \ell_{K\left(\eta, J_{1}\right)}$ and $\mathscr{B} \ell_{K\left(\eta, J_{2}\right)}$ are isomorphic forms. That is, there is a right $\mathbb{Z} \Gamma_{n}$-module isomorphism $\psi: \mathcal{A}_{n}\left(K\left(\eta, J_{1}\right)\right) \rightarrow \mathcal{A}_{n}\left(K\left(\eta, J_{2}\right)\right)$ such that for any $x, y \in \mathcal{A}_{n}\left(K\left(\eta, J_{1}\right)\right)$,

$$
\mathcal{B} \ell_{K\left(\eta, J_{1}\right)}(x, y)=\mathscr{B} \ell_{K\left(\eta, J_{2}\right)}(\psi(x), \psi(y)) .
$$

Using Theorem 3.4, we have a right $\mathbb{Z} \Gamma_{n}$-module isomorphism $\psi: H_{1}\left(E(K) ; \mathbb{Z} \Gamma_{n}\right) \oplus$ $H_{1}\left(E\left(J_{1}\right) ; \mathbb{Z} \Gamma_{n}\right) \rightarrow H_{1}\left(E(K) ; \mathbb{Z} \Gamma_{n}\right) \oplus H_{1}\left(E\left(J_{2}\right) ; \mathbb{Z} \Gamma_{n}\right)$. Since $H_{1}\left(E(K) ; \mathbb{Z} \Gamma_{n}\right)$ has no $\mathbb{Z} \Gamma_{n}^{\prime}$-torsion by Proposition 5.3 and $H_{1}\left(E\left(J_{i}\right) ; \mathbb{Z} \Gamma_{n}\right)$ is a $\mathbb{Z} \Gamma_{n}^{\prime}$-torsion module, for $i=1,2$, by Proposition 5.1, it follows that $\psi$ restricted to $H_{1}\left(E\left(J_{1}\right) ; \mathbb{Z} \Gamma_{n}\right)$ is a right $\mathbb{Z} \Gamma_{n}$-module isomorphism between $H_{1}\left(E\left(J_{1}\right) ; \mathbb{Z} \Gamma_{n}\right)$ and $H_{1}\left(E\left(J_{2}\right) ; \mathbb{Z} \Gamma_{n}\right)$. Hence if $x_{1}, y_{1} \in H_{1}\left(E\left(J_{1}\right) ; \mathbb{Z} \Gamma_{n}\right)$, then $\psi \circ i_{1}\left(x_{1}\right)=i_{2}\left(x_{2}\right)$ and $\psi \circ i_{1}\left(y_{1}\right)=i_{2}\left(y_{2}\right)$, for some $x_{2}, y_{2} \in H_{1}\left(E\left(J_{2}\right) ; \mathbb{Z} \Gamma_{n}\right)$. Finally, we have the following.:

$$
\begin{aligned}
\mathscr{B} \ell_{K\left(\eta, J_{1}\right)}^{\otimes}\left(x_{1}, y_{1}\right) & =\mathcal{B}_{K\left(\eta, J_{1}\right)}\left(i_{1}\left(x_{1}\right), i_{1}\left(y_{1}\right)\right) \quad \text { (by Theorem 4.6) } \\
& =\mathscr{B} \ell_{K\left(\eta, J_{2}\right)}\left(\psi\left(i_{1}\left(x_{1}\right)\right), \psi\left(i_{1}\left(y_{1}\right)\right)\right) \\
& =\mathscr{B} \ell_{K\left(\eta, J_{2}\right)}\left(i_{2}\left(x_{2}\right), i_{2}\left(y_{2}\right)\right) \\
& =\mathscr{B} \ell_{K\left(\eta, J_{2}\right)}^{\otimes}\left(x_{2}, y_{2}\right) .
\end{aligned}
$$

Therefore $\mathcal{B} \ell_{K\left(\eta, J_{1}\right)}^{\otimes}$ and $\mathcal{B} \ell_{K\left(\eta, J_{2}\right)}^{\otimes}$ are isomorphic forms. 


\section{Relating $\mathscr{B} \ell_{K(\eta, J)}^{\otimes}$ to the equivariant intersection form $\lambda_{J}$}

Our main result is that there exist knots with isomorphic $n$th higher-order Alexander modules, but non-isomorphic $n$th higher-order linking forms. The idea behind the construction of our examples is to infect the same knot $K$ along the same element $\eta \in$ $\pi_{1}(E(K))^{(n)}, \eta \notin \pi_{1}(E(K))^{(n+1)}$ by different knots $J_{1}$ and $J_{2}$ such that $\mathcal{A}_{0}\left(J_{1}\right) \cong$ $\mathcal{A}_{0}\left(J_{2}\right)$. Corollary 3.6 implies that the results of these infections will have isomorphic $i$-th order Alexander modules for $0 \leq i \leq n$. From Theorem 5.4, we know that if we choose $K$ to be a fibered knot, it suffices to find examples of knots $J_{1}$ and $J_{2}$ such that $\mathcal{A}_{0}\left(J_{1}\right) \cong \mathcal{A}_{0}\left(J_{2}\right)$, but $\mathscr{B} \ell_{K\left(\eta, J_{1}\right)}^{\otimes} \nsucceq \mathscr{B} \ell_{K\left(\eta, J_{2}\right)}^{\otimes}$. In this section, we will relate to $\mathscr{B} \ell_{K(\eta, J)}^{\otimes}$ a new linking form $\widehat{\mathscr{B}}_{K(\eta, J)}$ defined on the 0 -framed surgery on $S^{3}$ along $J$, which we will then relate to the equivariant intersection form, $\lambda_{J}$, on a particular 4-manifold, $W_{J}$, associated to $J$.

Let $M_{J}$ denote the closed 3-manifold resulting from 0-framed surgery on $S^{3}$ along $J$. The kernel of $\pi_{1}(E(J)) \rightarrow \pi_{1}\left(M_{J}\right)$ is normally generated by the longitude of $J$, which is in the kernel of $\pi_{1}(E(J)) \rightarrow \Gamma_{n}$. Hence $\pi_{1}(E(J)) \rightarrow \Gamma_{n}$ factors through $\pi_{1}\left(M_{J}\right)$, inducing a $\Gamma_{n}$ coefficient system on $M_{J}$.

Proposition 6.1. If $\eta \in \pi_{1}(E(K))^{(n)}$ and $\eta \notin \pi_{1}(E(K))^{(n+1)}$, then

$$
H_{1}\left(E(J) ; \mathbb{Z} \Gamma_{n}\right) \cong H_{1}\left(M_{J} ; \mathbb{Z} \Gamma_{n}\right) \text {. }
$$

Proof. We consider the Mayer-Vietoris sequence with $\mathbb{Z} \Gamma_{n}$ coefficients for $M_{J} \cong$ $E(J) \cup_{\partial E(J)}\left(D^{2} \times S^{1}\right)$,

$$
H_{1}\left(M_{J}\right) \stackrel{\partial_{*}}{\longrightarrow} H_{0}(\partial E(J)) \stackrel{\left(\psi_{1}, \psi_{2}\right)}{\longrightarrow} H_{0}(E(J)) \oplus H_{0}\left(D^{2} \times S^{1}\right) .
$$

By Lemma 3.3 we have that $\psi_{1}: H_{0}(\partial E(J)) \rightarrow H_{0}(E(J))$ is an isomorphism. Therefore $\partial_{*}: H_{1}\left(M_{J}\right) \rightarrow H_{0}(\partial E(J))$ is the trivial map. Thus we have the following:

$$
H_{1}(\partial E(J)) \stackrel{\left(\psi_{1}, \psi_{2}\right)}{\longrightarrow} H_{1}(E(J)) \oplus H_{1}\left(D^{2} \times S^{1}\right) \stackrel{j_{*}+k_{*}}{\longrightarrow} H_{1}\left(M_{J}\right) \longrightarrow 0 .
$$

By Lemma 3.3, we also have that $\psi_{1}: H_{0}(\partial E(J)) \rightarrow H_{0}(E(J))$ is the trivial map. Since $\eta \notin \pi_{1}(E(K))^{(n+1)}$ the generator of $\pi_{1}\left(D^{2} \times S^{1} ; \mathbb{Z}\right), \mu_{J}$, gets unwound in the $\Gamma_{n}$-cover. Hence $H_{1}\left(D^{2} \times S^{1}\right)=0$. Therefore, $j_{*}: H_{1}(E(J)) \rightarrow H_{1}\left(M_{J}\right)$ is an isomorphism.

Notice that since $H_{1}\left(E(J) ; \mathbb{Z} \Gamma_{n}\right)$ is a $\mathbb{Z} \Gamma_{n}$-torsion module by Proposition 4.2, it follows that $H_{1}\left(M_{J} ; \mathbb{Z} \Gamma_{n}\right)$ is as well. So the Bockstein map, $B: H_{2}\left(M_{J} ; \mathcal{K}_{n} / \mathbb{Z} \Gamma_{n}\right) \rightarrow$ $H_{1}\left(M_{J} ; \mathbb{Z} \Gamma_{n}\right)$, is an isomorphism by the same argument as in Section 4. 
Definition 6.2. If $\eta \in \pi_{1}(E(K))^{(n)}$ and $\eta \notin \pi_{1}(E(K))^{(n+1)}$, define $\widehat{\mathscr{B}}_{K(\eta, J)}$ : $H_{1}\left(M_{J} ; \mathbb{Z} \Gamma_{n}\right) \rightarrow H_{1}\left(M_{J} ; \mathbb{Z} \Gamma_{n}\right)^{\#}$ to be the composition of the following maps:

$$
\begin{aligned}
H_{1}\left(M_{J} ; \mathbb{Z} \Gamma_{n}\right) \stackrel{B^{-1}}{\longrightarrow} & H_{2}\left(M_{J} ; \mathcal{K}_{n} / \mathbb{Z} \Gamma_{n}\right) \\
\stackrel{P . D .}{\longrightarrow} & \overline{H^{1}\left(M_{J} ; \mathcal{K}_{n} / \mathbb{Z} \Gamma_{n}\right)} \stackrel{\kappa}{\longrightarrow} H_{1}\left(M_{J} ; \mathbb{Z} \Gamma_{n}\right)^{\#},
\end{aligned}
$$

where P.D. is the Poincare duality isomorphism and $\kappa$ is the Kronecker evaluation map.

Theorem 6.3. If $\eta \in \pi_{1}(E(K))^{(n)}$ and $\eta \notin \pi_{1}(E(K))^{(n+1)}$, then $B \ell_{K(\eta, J)}^{\otimes} \cong$ $\widehat{\mathcal{B}}_{K(\eta, J)}$. That is,

$$
\mathcal{B} \ell_{K(\eta, J)}^{\otimes}\left(x_{1}, y_{1}\right)=\widehat{\mathscr{B}}_{K(\eta, J)}\left(j_{*}\left(x_{1}\right), j_{*}\left(y_{1}\right)\right) .
$$

Proof. Since $j$ is an inclusion map, the proof is the same as that of the second claim in the proof of Theorem 4.6. Note however, that since $\partial M_{J}=\emptyset$, the map $\pi$ which appears in that proof is unnecessary.

Therefore, we have reduced our problem to finding examples of knots $J_{1}$ and $J_{2}$ such that $\mathcal{A}_{0}\left(J_{1}\right) \cong \mathcal{A}_{0}\left(J_{2}\right)$, but $\widehat{\mathscr{B} \ell}_{K\left(\eta, J_{1}\right)} \nsucceq \widehat{\mathscr{B} \ell}_{K\left(\eta, J_{2}\right)}$. To accomplish this, we will relate $\widehat{\mathscr{B}}_{K(\eta, J)}$ to the equivariant intersection form, $\lambda_{J}$, on a particular 4-manifold, $W_{J}$, associated to $J$.

Since the bordism group $\Omega_{3}\left(S^{1}\right)=0$, we can choose a 4-manifold $W_{J}$ which bounds $M_{J}$ and such that $\pi_{1}\left(W_{J}\right) \cong \mathbb{Z}$, generated by the meridian of $J$. Furthermore, by adding copies of $\pm \mathbb{C P}^{2}$, we can choose $W_{J}$ so that the signature of it is zero. (See [COT2].) Since the kernel of $\pi_{1}(E(J)) \rightarrow \pi_{1}\left(W_{J}\right)$ is $\pi_{1}(E(J))^{\prime}$, which is in the kernel of $\pi_{1}(E(J)) \rightarrow \Gamma_{n}$, it follows that $\pi_{1}(E(J)) \rightarrow \Gamma_{n}$ factors through $\pi_{1}\left(W_{J}\right)$, defining an induced $\Gamma_{n}$ coefficient system on $W_{J}$. Let $\lambda_{J}: H_{2}\left(W_{J} ; \mathbb{Z} \Gamma_{n}\right) \rightarrow$ $\operatorname{Hom}_{\mathbb{Z} \Gamma_{n}}\left(H_{2}\left(W_{J} ; \mathbb{Z} \Gamma_{n}\right), \mathbb{Z} \Gamma_{n}\right)$ be the equivariant intersection form on $W_{J}$ with $\mathbb{Z} \Gamma_{n}$ coefficients. That is, $\lambda_{J}$ is the composition of the following maps:

$$
\begin{aligned}
H_{2}\left(W_{J} ; \mathbb{Z} \Gamma_{n}\right) \stackrel{\pi}{\longrightarrow} H_{2}\left(W_{J}, M_{J} ; \mathbb{Z} \Gamma_{n}\right) \stackrel{P . D .}{\longrightarrow} \overline{H^{2}\left(W_{J} ; \mathbb{Z} \Gamma_{n}\right)} \\
\stackrel{\kappa}{\longrightarrow} \overline{\operatorname{Hom}_{\mathbb{Z} \Gamma_{n}}\left(H_{2}\left(W_{J} ; \mathbb{Z} \Gamma_{n}\right), \mathbb{Z} \Gamma_{n}\right) .}
\end{aligned}
$$

We recall the following definitions from $[R]$.

Definition 6.4 ([R], pp. 60-61, 145, 181, 242). Let $S$ be a right denominator set for a ring with involution, $R$, and let $\mathcal{M}$ be an $R$-module. A symmetric form over $R, \alpha: \mathcal{M} \rightarrow \overline{\operatorname{Hom}_{R}(\mathcal{M}, R)}$, is $S$-non-singular if $\alpha \otimes \mathrm{id}: \mathcal{M} \otimes_{R} R S^{-1} \rightarrow$ $\overline{\operatorname{Hom}_{R}(\mathcal{M}, R)} \otimes_{R} R S^{-1}$ is an $R S^{-1}$-module isomorphism. 
Definition 6.5 ([R], p. 243). The boundary of an $S$-non-singular symmetric form over $R, \alpha: \mathcal{M} \rightarrow \overline{\operatorname{Hom}_{R}(\mathcal{M}, R)} \equiv \mathcal{M}^{*}$, is the non-singular (even) symmetric linking form over $(R, S)$ defined by

$$
\begin{aligned}
\partial \alpha: \operatorname{coker} \alpha & \longrightarrow \overline{\operatorname{Hom}_{R}\left(\mathcal{M}^{*}, R S^{-1} / R\right)} \\
x & \longmapsto\left(y \longmapsto x(z) \cdot s^{-1}\right)
\end{aligned}
$$

for any $x, y \in \overline{\operatorname{Hom}_{R}(\mathcal{M}, R)} \equiv \mathcal{M}^{*}$, where $z \in \mathcal{M}, s \in S$ are chosen so that $y s=\alpha(z)$.

If $\eta \in \pi_{1}(E(K))^{(n)}$ and $\eta \notin \pi_{1}(E(K))^{(n+1)}$, we will show in Proposition 6.6 that the equivariant intersection form $\lambda_{J}$, as above, is a $\left(\mathbb{Z} \Gamma_{n}-\{0\}\right)$-non-singular symmetric form over $\mathbb{Z} \Gamma_{n}$. Furthermore, we will show in Theorem 6.9 that $\partial \lambda_{J} \cong \widehat{\mathscr{B}}_{K(\eta, J)}$. In Section 7, this will allow us to fit $\lambda_{J}$ and $\widehat{\mathcal{B} \ell} K_{(\eta, J)}$ into an exact sequence of Witt groups. The result will be that we can distinguish $\widehat{\mathscr{B}} \ell_{K}\left(\eta, J_{1}\right)$ from $\widehat{\mathcal{B}}_{K\left(\eta, J_{2}\right)}$ by using an invariant of $\lambda_{J_{1}}$ and $\lambda J_{2}$.

Proposition 6.6. If $\eta \in \pi_{1}(E(K))^{(n)}$ and $\eta \notin \pi_{1}(E(K))^{(n+1)}$, the equivariant intersection form $\lambda_{J}$, as above, is a $\left(\mathbb{Z} \Gamma_{n}-\{0\}\right)$-non-singular symmetric form over $\mathbb{Z} \Gamma_{n}$.

Proof. In order to prove the proposition, we require Lemmas 6.7 and 6.8 .

Lemma 6.7. $H_{p}\left(M_{J} ; \mathbb{Z} \Gamma_{n}\right) \cong H_{p}\left(M_{J} ; \mathbb{Z}\left[t, t^{-1}\right]\right) \bigotimes_{\mathbb{Z}\left[t, t^{-1}\right]} \mathbb{Z} \Gamma_{n}$ and $H_{p}\left(W_{J} ; \mathbb{Z} \Gamma_{n}\right) \cong$ $H_{p}\left(W_{J} ; \mathbb{Z}\left[t, t^{-1}\right]\right) \bigotimes_{\mathbb{Z}\left[t, t^{-1}\right]} \mathbb{Z} \Gamma_{n}$ as right $\mathbb{Z} \Gamma_{n}$-modules.

Proof. If $\widetilde{M_{J}}$ is the universal cover of $M_{J}$, we have the following:

$$
\begin{aligned}
H_{p}\left(M_{J} ; \mathbb{Z} \Gamma_{n}\right)= & H_{p}\left(C_{*}\left(\widetilde{M_{J}}\right) \otimes_{\mathbb{Z} \pi_{1}\left(M_{J}\right)} \mathbb{Z} \Gamma_{n}\right) \\
\cong & H_{p}\left(C_{*}\left(\widetilde{M_{J}}\right) \otimes_{\mathbb{Z} \pi_{1}\left(M_{J}\right)} \mathbb{Z}\left[t, t^{-1}\right] \otimes_{\mathbb{Z}\left[t, t^{-1}\right]} \mathbb{Z} \Gamma_{n}\right) \quad([\text { HS, p. 109] }) \\
\cong & H_{p}\left(C_{*}\left(\widetilde{M_{J}}\right) \otimes_{\mathbb{Z} \pi_{1}\left(M_{J}\right)} \mathbb{Z}\left[t, t^{-1}\right]\right) \otimes_{\mathbb{Z}\left[t, t^{-1}\right]} \mathbb{Z} \Gamma_{n} \\
& \text { since } \mathbb{Z} \Gamma_{n} \text { is a free, hence flat, } \mathbb{Z}\left[t, t^{-1}\right] \text { module [P, Lemma 1.3] } \\
\cong & H_{p}\left(M_{J} ; \mathbb{Z}\left[t, t^{-1}\right]\right) \otimes_{\mathbb{Z}\left[t, t^{-1}\right]} \mathbb{Z} \Gamma_{n} .
\end{aligned}
$$

A similar argument holds for $W_{J}$.

Lemma 6.8. $\kappa: H^{2}\left(W_{J} ; \mathbb{Z} \Gamma_{n}\right) \rightarrow \operatorname{Hom}_{\mathbb{Z} \Gamma_{n}}\left(H_{2}\left(W_{J} ; \mathbb{Z} \Gamma_{n}\right), \mathbb{Z} \Gamma_{n}\right)$ is a $\mathbb{Z} \Gamma_{n}$-module isomorphism.

Proof. As $\pi_{1}\left(W_{J}\right)$ is generated by the meridian of $J$, which is identified to the longitude of $\eta$ in $E(K(\eta, J))$, and $\eta$ gets unwound in the $\mathbb{Z} \Gamma_{n}$-cover, $H_{1}\left(W_{J} ; \mathbb{Z} \Gamma_{n}\right)=0$. 
By analyzing the Universal Coefficient Spectral Sequence [Le, Thm. 2.3] we have the following exact sequence:

$$
\begin{aligned}
& \operatorname{Ext}_{\mathbb{Z} \Gamma_{n}}^{2}\left(H_{0}\left(W_{J} ; \mathbb{Z} \Gamma_{n}\right), \mathbb{Z} \Gamma_{n}\right) \longrightarrow H^{2}\left(W_{J} ; \mathbb{Z} \Gamma_{n}\right) \\
& \quad \stackrel{\kappa}{\longrightarrow} \operatorname{Hom}_{\mathbb{Z} \Gamma_{n}}\left(H_{2}\left(W_{J} ; \mathbb{Z} \Gamma_{n}\right), \mathbb{Z} \Gamma_{n}\right) \longrightarrow \operatorname{Ext}_{\mathbb{Z} \Gamma_{n}}^{3}\left(H_{0}\left(W_{J} ; \mathbb{Z} \Gamma_{n}\right), \mathbb{Z} \Gamma_{n}\right) .
\end{aligned}
$$

By Lemma 6.7, $H_{0}\left(W_{J} ; \mathbb{Z} \Gamma_{n}\right) \cong H_{0}\left(W_{J} ; \mathbb{Z}\left[t, t^{-1}\right]\right) \bigotimes_{\mathbb{Z}\left[t, t^{-1}\right]} \mathbb{Z} \Gamma_{n}$. Since the homological dimension of $H_{0}\left(W_{J} ; \mathbb{Z}\left[t, t^{-1}\right]\right)$ is 1 , and since $\mathbb{Z} \Gamma_{n}$ is a free, and therefore flat, $\mathbb{Z}\left[t, t^{-1}\right]$-module, $H_{0}\left(W_{J} ; \mathbb{Z}\left[t, t^{-1}\right]\right) \otimes_{\mathbb{Z}\left[t, t^{-1}\right]} \mathbb{Z} \Gamma_{n}$ also has homological dimension 1. Therefore $\operatorname{Ext}_{\mathbb{Z} \Gamma_{n}}^{p}\left(H_{0}\left(W_{J} ; \mathbb{Z} \Gamma_{n}\right), \mathbb{Z} \Gamma_{n}\right)=0$ for $p=2,3$. Hence $\kappa: H^{2}\left(W_{J} ; \mathbb{Z} \Gamma_{n}\right) \rightarrow \operatorname{Hom}_{\mathbb{Z} \Gamma_{n}}\left(H_{2}\left(W_{J} ; \mathbb{Z} \Gamma_{n}\right), \mathbb{Z} \Gamma_{n}\right)$ is a $\mathbb{Z} \Gamma_{n}$-module isomorphism.

Having proven these lemmas, we continue our proof of Proposition 6.6. Since $\lambda_{J} \cong \kappa \circ P . D . \circ \pi$, it remains to be shown that $\pi \otimes \mathrm{id}, P . D . \otimes \mathrm{id}$, and $\kappa \otimes \mathrm{id}$ are $\mathcal{K}_{n}$-module isomorphisms.

Since $\mathcal{K}_{n}$ is a flat $\mathbb{Z} \Gamma_{n}$-module, the following is an exact sequence of $\mathcal{K}_{n}$-modules:

$$
\begin{aligned}
& H_{2}\left(M_{J} ; \mathbb{Z} \Gamma_{n}\right) \otimes_{\mathbb{Z} \Gamma_{n}} \mathcal{K}_{n} \longrightarrow H_{2}\left(W_{J} ; \mathbb{Z} \Gamma_{n}\right) \otimes_{\mathbb{Z} \Gamma_{n}} \mathcal{K}_{n} \\
& \quad \stackrel{\pi \otimes \mathrm{id}}{\longrightarrow} H_{2}\left(W_{J}, M_{J} ; \mathbb{Z} \Gamma_{n}\right) \otimes_{\mathbb{Z} \Gamma_{n}} \mathcal{K}_{n} \longrightarrow H_{1}\left(M_{J} ; \mathbb{Z} \Gamma_{n}\right) \otimes_{\mathbb{Z} \Gamma_{n}} \mathcal{K}_{n} .
\end{aligned}
$$

By Propositions 4.2 and 6.1, $H_{1}\left(M_{J} ; \mathbb{Z} \Gamma_{n}\right) \otimes_{\mathbb{Z} \Gamma_{n}} \mathcal{K}_{n}=0$. Again by the flatness of $\mathcal{K}_{n}, H_{2}\left(M_{J} ; \mathbb{Z} \Gamma_{n}\right) \otimes_{\mathbb{Z} \Gamma_{n}} \mathcal{K}_{n} \cong H_{2}\left(M_{J} ; \mathcal{K}_{n}\right)$. By Poincaré Duality and the Universal Coefficient Theorem, $H_{2}\left(M_{J} ; \mathcal{K}_{n}\right) \cong \operatorname{Hom}_{\mathbb{Z} \Gamma_{n}}\left(H_{1}\left(M_{J} ; \mathbb{Z} \Gamma_{n}\right), \mathcal{K}_{n}\right)$. But since $H_{1}\left(M_{J} ; \mathbb{Z} \Gamma_{n}\right)$ is a torsion-module, $\operatorname{Hom}_{\mathbb{Z} \Gamma_{n}}\left(H_{1}\left(M_{J} ; \mathbb{Z} \Gamma_{n}\right), \mathcal{K}_{n}\right)=0$. Hence $\pi \otimes \mathrm{id}$ is a $\mathcal{K}_{n}$-module isomorphism.

By Poincaré Duality and Lemma 6.8, it follows that P.D. $\otimes$ id and $\kappa \otimes$ id are $\mathcal{K}_{n}$-module isomorphisms. Therefore,

$$
\lambda_{J} \otimes \mathrm{id}: H_{2}\left(W ; \mathbb{Z} \Gamma_{n}\right) \otimes_{\mathbb{Z} \Gamma_{n}} \mathcal{K}_{n} \longrightarrow \overline{\operatorname{Hom}_{\mathbb{Z} \Gamma_{n}}\left(H_{2}\left(W ; \mathbb{Z} \Gamma_{n}\right), \mathbb{Z} \Gamma_{n}\right) \otimes_{\mathbb{Z} \Gamma_{n}} \mathcal{K}_{n}}
$$

is a $\mathcal{K}_{n}$-module isomorphism. Thus, $\lambda_{J}$ is a $\left(\mathbb{Z} \Gamma_{n}-\{0\}\right)$-non-singular symmetric form over $\mathbb{Z} \Gamma_{n}$.

Theorem 6.9. If $\eta \in \pi_{1}(E(K))^{(n)}$ and $\eta \notin \pi_{1}(E(K))^{(n+1)}$, it follows that $\partial \lambda_{J} \cong$ $\widehat{\mathscr{B}}_{K(\eta, J)}: H_{1}\left(M_{J} ; \mathbb{Z} \Gamma_{n}\right) \rightarrow H_{1}\left(M_{J} ; \mathbb{Z} \Gamma_{n}\right)^{\#}$.

Proof. By definition, $\partial \lambda_{J}$ is defined as follows:

$$
\begin{aligned}
\partial \lambda_{J}: \operatorname{coker} \lambda_{J} & \longrightarrow\left(\overline{\operatorname{Hom}_{\mathbb{Z} \Gamma_{n}}\left(H_{2}\left(W_{J} ; \mathbb{Z} \Gamma_{n}\right), \mathbb{Z} \Gamma_{n}\right)}\right)^{\#} \\
x & \longmapsto\left(y \longmapsto x(z) \cdot \gamma^{-1}\right)
\end{aligned}
$$


for any $x, y \in \overline{\operatorname{Hom}_{\mathbb{Z} \Gamma_{n}}\left(H_{2}\left(W_{J} ; \mathbb{Z} \Gamma_{n}\right), \mathbb{Z} \Gamma_{n}\right)}$, where $z \in H_{2}\left(W_{J} ; \mathbb{Z} \Gamma_{n}\right)$ and $\gamma \in$ $\mathbb{Z} \Gamma_{n}-\{0\}$ are chosen so that $y \gamma=\lambda_{J}(z)$.

Consider the following commutative diagram.

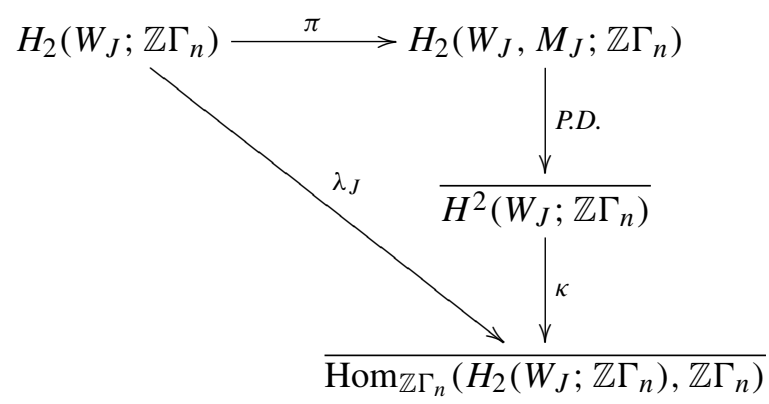

Recall from Lemma 6.8 that P.D. and $\kappa$ are $\mathbb{Z} \Gamma_{n}$-module isomorphisms. Since $\lambda_{J}=$ $\kappa \circ P . D . \circ \pi$, it follows that coker $\lambda_{J}=(\kappa \circ P . D$. $)(\operatorname{coker} \pi)$.

We define $\psi$ to be the following:

$$
\begin{aligned}
\psi: \operatorname{coker} \pi & \longrightarrow H_{2}\left(W_{J}, M_{J} ; \mathbb{Z} \Gamma_{n}\right)^{\#} \\
a & \longmapsto\left(b \longmapsto[(\kappa \circ P . D .)(a)](z) \cdot \gamma^{-1}\right)
\end{aligned}
$$

for any $a, b \in H_{2}\left(W_{J}, M_{J} ; \mathbb{Z} \Gamma_{n}\right)$, where $z \in H_{2}\left(W_{J} ; \mathbb{Z} \Gamma_{n}\right), \gamma \in \mathbb{Z} \Gamma_{n}$ are chosen so that $\pi(z)=b \gamma$.

Lemma 6.10. $\partial \lambda_{J}$ is isomorphic to $\psi$ under $\kappa \circ P . D .$. That is, $\psi=(\kappa \circ P . D .)^{\#} \circ$ $\partial \lambda_{J} \circ(\kappa \circ P . D$.$) .$

Proof. In order to show this, we must show that the following diagram commutes.

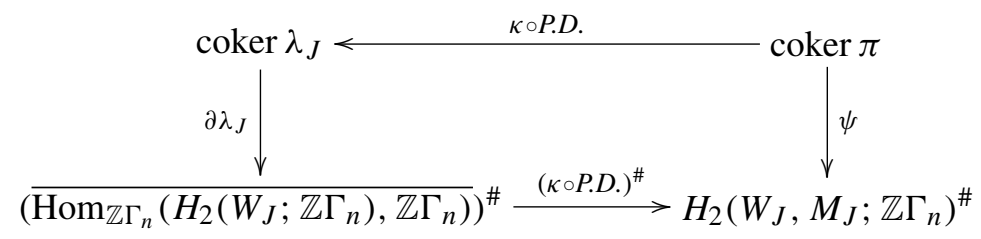

Suppose $a, b \in H_{2}\left(W_{J}, M_{J} ; \mathbb{Z} \Gamma_{n}\right)$ and $z \in H_{2}\left(W_{J} ; \mathbb{Z} \Gamma_{n}\right), \gamma \in \mathbb{Z} \Gamma_{n}$ are chosen so that $\pi(z)=b \gamma$. Let $x=(\kappa \circ P . D).(a)$ and $y=(\kappa \circ P . D$. $)(b)$. Then $\lambda_{J}(z)=$ $(\kappa \circ P . D . \circ \pi)(z)=(\kappa \circ P . D).(b \gamma)=y \gamma$. By definition, $\left[\left(\partial \lambda_{J}\right)(x)\right](y)=x(z) \gamma^{-1}$. 
Therefore,

$$
\begin{aligned}
{\left[\left[(\kappa \circ P . D .)^{\#} \circ \partial \lambda_{J} \circ(\kappa \circ P . D .)\right](a)\right](b) } & =\left[\left(\partial \lambda_{J} \circ \kappa \circ P . D .\right)(a)\right]((\kappa \circ P . D .)(b)) \\
& =\left[\partial \lambda_{J}(x)\right](y) \\
& =x(z) \gamma^{-1} \\
& =[(\kappa \circ P . D .)(a)](z) \gamma^{-1} \\
& =[\psi(a)](b) .
\end{aligned}
$$

Hence $\partial \lambda_{J}$ is isomorphic to $\psi$.

In order to relate $\psi$ to $\widehat{\mathscr{B}}_{K(\eta, J)}$, it will be easier to work with a more algebraic definition of $\psi$. We begin by proving the following lemma.

Lemma 6.11. The mapping

$$
\pi^{\#}: \overline{\operatorname{Hom}_{\mathbb{Z} \Gamma_{n}}\left(H_{2}\left(W_{J}, M_{J} ; \mathbb{Z} \Gamma_{n}\right), \mathcal{K}_{n}\right)} \longrightarrow \overline{\operatorname{Hom}_{\mathbb{Z} \Gamma_{n}}\left(H_{2}\left(W_{J} ; \mathbb{Z} \Gamma_{n}\right), \mathcal{K}_{n}\right)}
$$

is an isomorphism.

Proof. In the proof of Proposition 6.6, we showed that $\pi \otimes \mathrm{id}: H_{2}\left(W_{J} ; \mathbb{Z} \Gamma_{n}\right) \otimes_{\mathbb{Z} \Gamma_{n}}$ $\mathcal{K}_{n} \rightarrow H_{2}\left(W_{J}, M_{J} ; \mathbb{Z} \Gamma_{n}\right) \otimes_{\mathbb{Z} \Gamma_{n}} \mathcal{K}_{n}$ is a $\mathcal{K}_{n}$-module isomorphism. Since $\mathcal{K}_{n}$ is a flat $\mathbb{Z} \Gamma_{n}$-module, by Poincaré Duality and the Universal Coefficient Theorem,

$$
\pi^{\#}: \overline{\operatorname{Hom}_{\mathbb{Z} \Gamma_{n}}\left(H_{2}\left(W_{J}, M_{J} ; \mathbb{Z} \Gamma_{n}\right), \mathcal{K}_{n}\right)} \longrightarrow \overline{\operatorname{Hom}_{\mathbb{Z} \Gamma_{n}}\left(H_{2}\left(W_{J} ; \mathbb{Z} \Gamma_{n}\right), \mathcal{K}_{n}\right)}
$$

is an isomorphism.

By definition, $[\psi(a)](b)=[(\kappa \circ P . D).(a)](z) \cdot \gamma^{-1}$ where $\pi(z)=b \gamma$. So $\left[\pi^{\#}(\psi(a))\right](z)=[\psi(a)](\pi(z))=[\psi(a)](b \gamma)=[(\kappa \circ P . D).(a)](z)$. Hence we have that $\psi$ is the composition of the following maps:

$$
\begin{aligned}
& H_{2}\left(W_{J}, M_{J} ; \mathbb{Z} \Gamma_{n}\right) \stackrel{P . D .}{\longrightarrow} \overline{H^{2}\left(W_{J} ; \mathbb{Z} \Gamma_{n}\right)} \\
& \stackrel{\kappa}{\longrightarrow} \overline{\operatorname{Hom}_{\mathbb{Z} \Gamma_{n}}\left(H_{2}\left(W_{J} ; \mathbb{Z} \Gamma_{n}\right), \mathbb{Z} \Gamma_{n}\right)} \\
& \stackrel{\operatorname{Hom}_{\mathbb{Z} \Gamma_{n}}\left(H_{2}\left(W_{J} ; \mathbb{Z} \Gamma_{n}\right), \mathcal{K}_{n}\right)}{\longrightarrow} \overline{\operatorname{Hom}_{\mathbb{Z} \Gamma_{n}}\left(H_{2}\left(W_{J}, M_{J} ; \mathbb{Z} \Gamma_{n}\right), \mathcal{K}_{n}\right)} \\
& \longrightarrow H_{2}\left(W_{J}, M_{J} ; \mathbb{Z} \Gamma_{n}\right)^{\#} .
\end{aligned}
$$

Recall that since $\pi_{1}\left(W_{J}\right)$ is generated by the meridian of $J$, which is identified to the longitude of $\eta$ in $E(K(\eta, J))$, and $\eta$ gets unwound in the $\mathbb{Z} \Gamma_{n}$-cover, it follows that $H_{1}\left(W_{J} ; \mathbb{Z} \Gamma_{n}\right)=0$. Therefore, the following is an exact sequence:

$$
H_{2}\left(W_{J} ; \mathbb{Z} \Gamma_{n}\right) \stackrel{\pi}{\longrightarrow} H_{2}\left(W_{J}, M_{J} ; \mathbb{Z} \Gamma_{n}\right) \stackrel{\partial_{*}}{\longrightarrow} H_{1}\left(M_{J} ; \mathbb{Z} \Gamma_{n}\right) \longrightarrow 0 .
$$


Hence coker $\pi \equiv \frac{H_{2}\left(W_{J}, M_{J} ; \mathbb{Z} \Gamma_{n}\right)}{\operatorname{im} \pi}=\frac{H_{2}\left(W_{J}, M_{J} ; \mathbb{Z} \Gamma_{n}\right)}{\operatorname{ker} \partial_{*}} \cong H_{1}\left(M_{J} ; \mathbb{Z} \Gamma_{n}\right)$. The following lemma will complete the proof of the theorem.

Lemma 6.12. $\psi$ is isomorphic to $\widehat{\mathscr{B}}_{K(\eta, J)}$. That is, $\psi=\partial_{*}^{\#} \circ \widehat{\mathscr{B}}_{K(\eta, J)} \circ \partial_{*}$.

Proof. Recall that $\widehat{\mathbb{B} \ell}_{K(\eta, J)}=B^{-1} \circ P . D . \circ \kappa$, where $B: H_{2}\left(M_{J} ; \mathcal{K}_{n} / \mathbb{Z} \Gamma_{n}\right) \rightarrow$ $H_{1}\left(M_{J} ; \mathbb{Z} \Gamma_{n}\right)$. Alternatively, we have $\widehat{\mathscr{B}}_{K(\eta, J)}=P . D . \circ C^{-1} \circ \kappa$ since the following is a commutative diagram of $\mathbb{Z} \Gamma_{n}$-module isomorphisms.

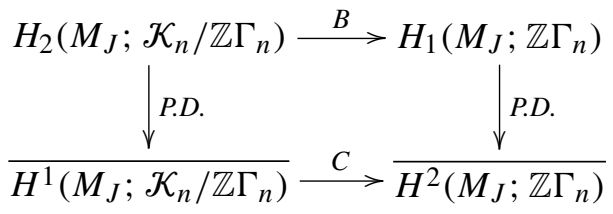

We must show that the following diagram commutes.

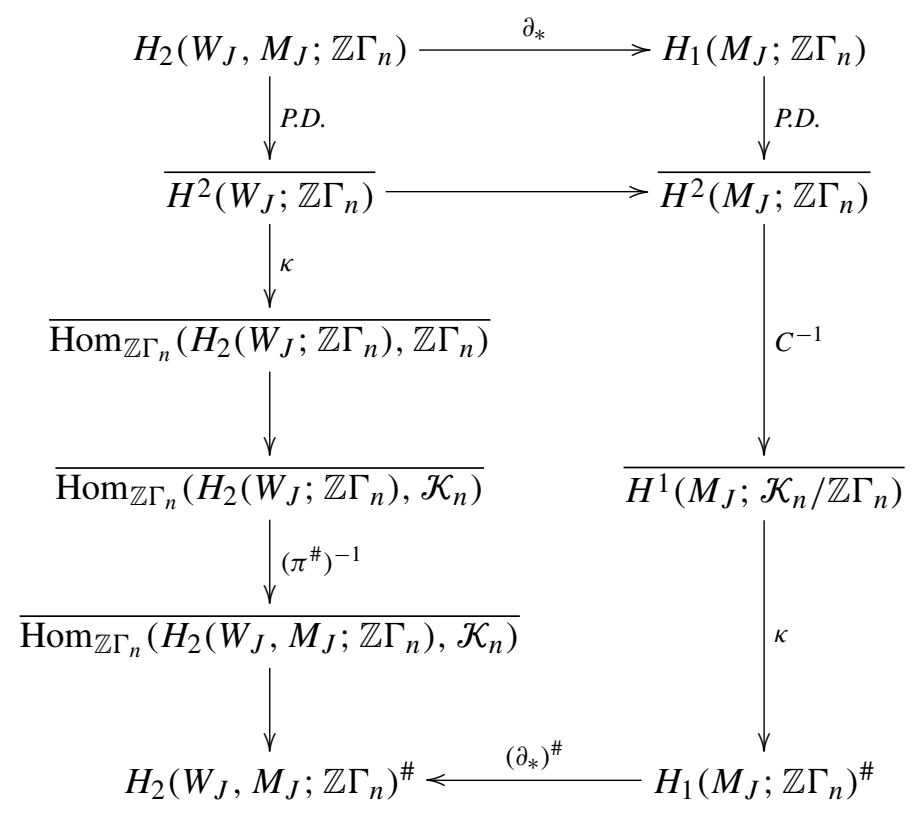

The top box commutes by the naturality of Poincaré Duality.

The short exact sequence of $\mathbb{Z} \Gamma_{n}$ chain groups

$$
0 \longrightarrow C_{*}\left(M_{J}\right) \longrightarrow C_{*}\left(W_{J}\right) \longrightarrow C_{*}\left(W_{J}, M_{J}\right) \longrightarrow 0
$$


gives rise to the following exact sequences:

$$
\begin{aligned}
& 0 \longrightarrow C_{*}\left(W_{J}, M_{J}\right)^{*} \longrightarrow C_{*}\left(W_{J}\right)^{*} \longrightarrow C_{*}\left(M_{J}\right)^{*} \\
& 0 \longrightarrow C_{*}\left(W_{J}, M_{J}\right)^{\circ} \longrightarrow C_{*}\left(W_{J}\right)^{\circ} \longrightarrow C_{*}\left(M_{J}\right)^{\circ} \\
& 0 \longrightarrow C_{*}\left(W_{J}, M_{J}\right)^{\#} \longrightarrow C_{*}\left(W_{J}\right)^{\#} \longrightarrow C_{*}\left(M_{J}\right)^{\#} .
\end{aligned}
$$

Here

$$
\begin{aligned}
\mathcal{M}^{*} & \equiv \overline{\operatorname{Hom}_{\mathbb{Z} \Gamma_{n}}\left(\mathcal{M}, \mathbb{Z} \Gamma_{n}\right)}, \\
\mathcal{M}^{\circ} & \equiv \overline{\operatorname{Hom}_{\mathbb{Z} \Gamma_{n}}\left(\mathcal{M}, \mathcal{K}_{n}\right)},
\end{aligned}
$$

and

$$
\mathcal{M}^{\#} \equiv \overline{\operatorname{Hom}_{\mathbb{Z} \Gamma_{n}}\left(\mathcal{M}, \mathcal{K}_{n} / \mathbb{Z} \Gamma_{n}\right)} .
$$

Also the short exact sequence $0 \rightarrow \mathbb{Z} \Gamma_{n} \rightarrow \mathcal{K}_{n} \rightarrow \mathcal{K}_{n} / \mathbb{Z} \Gamma_{n} \rightarrow 0$ gives rise to the following exact sequences:

$$
\begin{aligned}
& 0 \longrightarrow C_{*}\left(M_{J}\right)^{*} \longrightarrow C_{*}\left(M_{J}\right)^{\circ} \longrightarrow C_{*}\left(M_{J}\right)^{\#} \\
& 0 \longrightarrow C_{*}\left(W_{J}\right)^{*} \longrightarrow C_{*}\left(W_{J}\right)^{\circ} \longrightarrow C_{*}\left(W_{J}\right)^{\#} \\
& 0 \longrightarrow C_{*}\left(W_{J}, M_{J}\right)^{*} \longrightarrow C_{*}\left(W_{J}, M_{J}\right)^{\circ} \longrightarrow C_{*}\left(W_{J}, M_{J}\right)^{\#} .
\end{aligned}
$$

The lemma now follows from the commutativity of the following diagram.

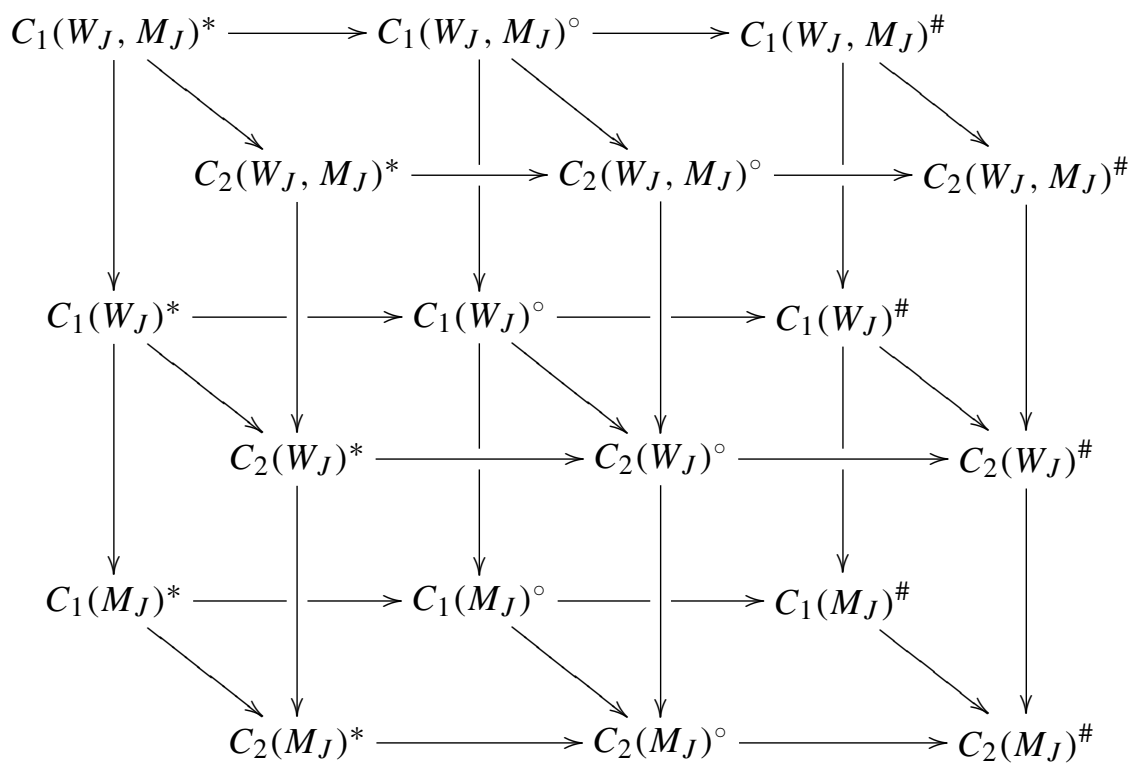




\section{L-theory and the $L^{2}$-signature}

If $R$ is a ring with involution and $S$ is a right denominator set for $R$, then from [R, pp. 172, 274] we have the following exact sequence of Witt groups:

$$
L(R) \longrightarrow L_{S}\left(R S^{-1}\right) \stackrel{\partial}{\longrightarrow} L(R, S) .
$$

Here $L(R)$ is the Witt group of nonsingular symmetric forms over $R ; L_{S}\left(R S^{-1}\right)$ is the Witt group of $S$-nonsingular symmetric forms over $R$; and $L(R, S)$ is the Witt group of non-singular symmetric linking forms over $(R, S)$.

Recall that we have reduced our problem to finding examples of knots $J_{1}$ and $J_{2}$ such that $\mathcal{A}_{0}\left(J_{1}\right) \cong \mathcal{A}_{0}\left(J_{2}\right)$, but $\widehat{\mathscr{B}}_{K\left(\eta, J_{1}\right)} \not \widehat{\mathcal{B}}_{K}\left(\eta, J_{2}\right)$. If $\eta \in \pi_{1}(E(K))^{(n)}$ and $\eta \notin \pi_{1}(E(K))^{(n+1)}$, we showed in Proposition 6.6, that $\lambda_{J} \in L_{\left(\mathbb{Z} \Gamma_{n}-\{0\}\right)}\left(\mathcal{K}_{n}\right)$, and in Theorem 6.9, that $\partial \lambda_{J} \cong \widehat{\mathcal{B}}_{K(\eta, J)}$. Hence we need an invariant defined on $L_{\left(\mathbb{Z} \Gamma_{n}-\{0\}\right)}\left(\mathcal{K}_{n}\right)$ that is trivial on the image of $L\left(\mathbb{Z} \Gamma_{n}\right)$. In this section, we will find that the desired invariant is the reduced $\mathrm{L}^{2}$-signature. Furthermore, we will observe that in our case, the reduced $\mathrm{L}^{2}$-signature of $\lambda_{J}$ is dependent only on the LevineTristram signatures of $J$. We refer the reader to Section 5 of [COT1] and Sections 2 and 5 of [COT2] for more details about the $\mathrm{L}^{2}$-signature.

Proposition 7.1 ([COT1], Cor. 5.7, Prop. 5.12). The $L^{2}$-signature $\sigma_{\Gamma}^{(2)}$ is a real valued homomorphism on the Witt group of nonsingular symmetric forms over $\mathcal{K}_{n}$, $L\left(\mathcal{K}_{n}\right)$. Furthermore, the $L^{2}$-signature equals the ordinary signature $\sigma_{0}$ on the image of $L\left(\mathbb{Z} \Gamma_{n}\right)$.

Therefore $\sigma_{\Gamma}^{(2)}-\sigma_{0}$ satisfies the desired conditions for our invariant. So we have the following definition.

Definition 7.2. The reduced $L^{2}$-signature of $\left(W_{J}, \Gamma_{n}\right)$, is defined to be

$$
\sigma_{\Gamma}^{(2)}\left(\lambda\left(W_{J}\right)\right)-\sigma_{0}\left(\lambda\left(W_{J}\right)\right),
$$

where $\lambda\left(W_{J}\right): H_{2}\left(W_{J} ; \mathbb{Z} \Gamma_{n}\right) \rightarrow \overline{\operatorname{Hom}_{\mathbb{Z} \Gamma_{n}}\left(H_{2}\left(W_{J} ; \mathbb{Z} \Gamma_{n}\right), \mathbb{Z} \Gamma_{n}\right)}$ is the equivariant intersection form on $W_{J}$ with $\mathbb{Z} \Gamma_{n}$ coefficients.

By Proposition 7.1, the reduced $\mathrm{L}^{2}$-signature is a well-defined real valued homomorphism on $L\left(\mathcal{K}_{n}\right)$. Furthermore, the reduced $\mathrm{L}^{2}$-signature is zero on the image of $L\left(\mathbb{Z} \Gamma_{n}\right)$. Therefore, it suffices to choose knots $J_{1}$ and $J_{2}$ such that $\mathcal{A}_{0}\left(J_{1}\right) \cong \mathcal{A}_{0}\left(J_{2}\right)$, but with reduced $\mathrm{L}^{2}$-signatures of $\left(W_{J_{1}}, \Gamma_{n}\right)$ and $\left(W_{J_{2}}, \Gamma_{n}\right)$ that are not equal.

Proposition 7.3 ([COT1], Prop. 5.13). If $\phi: \pi_{1}(W) \rightarrow \Gamma$ factors through a subgroup $\Sigma$, then $\sigma_{\Gamma}^{(2)}(\lambda(W))=\sigma_{\Sigma}^{(2)}(\lambda(W))$. 
Corollary 7.4. If $\eta \in \pi_{1}(E(K))^{(n)}$ and $\eta \notin \pi_{1}(E(K))^{(n+1)}$, the reduced $L^{2}$-signatures of $\left(W_{J}, \Gamma_{n}\right)$ and $\left(W_{J}, \mathbb{Z}\right)$ are equal.

Proof. Recall that our coefficient system on $W$ is defined by $\varphi$ in the following commutative diagram.

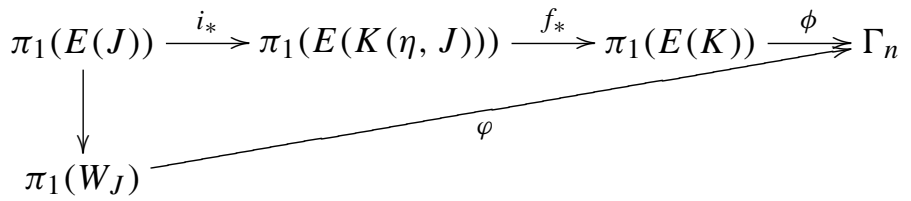

Recall also that $\pi_{1}\left(W_{J}\right) \cong \mathbb{Z}$ generated by a meridian of $J$, which is identified in $E(K(\eta, J))$ to $\eta$. Since we are assuming that $\eta \in \pi_{1}(E(K))^{(n)}$ and $\eta \notin \pi_{1}(E(K))^{(n+1)}, \varphi$ is a monomorphism. Therefore, $\varphi: \pi_{1}\left(W_{J}\right) \rightarrow \Gamma_{n}$ factors through $\mathbb{Z}$. It follows from Proposition 7.3 that $\sigma_{\Gamma_{n}}^{(2)}\left(\lambda\left(W_{J}\right)\right)=\sigma_{\mathbb{Z}}^{(2)}\left(\lambda\left(W_{J}\right)\right)$. Hence the reduced $\mathrm{L}^{2}$-signatures of $\left(W_{J}, \Gamma_{n}\right)$ and $\left(W_{J}, \mathbb{Z}\right)$ are equal.

Proposition 7.5 ([COT1], Prop 5.1, Lemma 5.9(4)). If $\eta \in \pi_{1}(E(K))^{(n)}$ and $\eta \notin$ $\pi_{1}(E(K))^{(n+1)}$, the reduced $L^{2}$-signature of $\left(W_{J}, \mathbb{Z}\right)$ is equal to the integral of the Levine-Tristram signatures of $J$, integrated over the circle of unit length.

Thus we have our main theorem:

Theorem 7.6. Given any $n \geq 1$, suppose $K$ is a fibered knot, that is not the unknot, and choose $\eta$ such that $\eta \in \pi_{1}(E(K))^{(n)}$ and $\eta \notin \pi_{1}(E(K))^{(n+1)}$. Let $J$ be a knot such that the integral of the Levine-Tristram signatures of $J$, integrated over the circle of unit length, is non-zero. Then $\mathcal{A}_{i}(K(\eta, J)) \cong \mathcal{A}_{i}(K(\eta,-J))$ for $0 \leq$ $i \leq n$ and $\mathscr{B} \ell_{i}(K(\eta, J)) \cong \mathscr{B} \ell_{i}(K(\eta,-J))$ for $0 \leq i<n$, but $\mathscr{B} \ell_{n}(K(\eta, J)) \varsubsetneqq$ $\mathscr{B} \ell_{n}(K(\eta,-J))$.

Proof. By Corollary 3.2, for $0 \leq i \leq n-1, \mathcal{A}_{i}(K(\eta, J)) \cong \mathcal{A}_{i}(K) \cong \mathcal{A}_{i}(K(\eta,-J))$. Furthermore, by Corollary 3.6, since the classical Alexander modules of $J$ and $-J$ are isomorphic, $\mathcal{A}_{n}(K(\eta, J)) \cong \mathcal{A}_{n}(K) \oplus\left(\mathcal{A}_{0}(J) \otimes_{\mathbb{Z}\left[t, t^{-1}\right]} \mathbb{Z} \Gamma_{n}\right) \cong \mathcal{A}_{n}(K) \oplus$ $\left(\mathcal{A}_{0}(-J) \otimes_{\mathbb{Z}\left[t, t^{-1}\right]} \mathbb{Z} \Gamma_{n}\right) \cong \mathcal{A}_{n}(K(\eta,-J))$.

By Theorem 4.1, for $0 \leq i \leq n-1, \mathscr{B} \ell_{i}(K(\eta, J)) \cong \mathscr{B} \ell_{i}(K) \cong \mathscr{B} \ell_{i}(K(\eta,-J))$. Now suppose $\mathscr{B} \ell_{n}(K(\eta, J))$ and $\mathscr{B} \ell_{n}(K(\eta,-J))$ are isomorphic. Since $K$ is a nontrivial fibered knot, it follows from Theorem 5.4, that $\mathscr{B} \ell_{K(\eta, J)}^{\otimes}$ and $\mathcal{B} \ell_{K(\eta,-J)}^{\otimes}$ are isomorphic, and therefore $\widehat{\mathscr{B}}_{K(\eta, J)} \cong \widehat{\mathscr{B}}_{K(\eta,-J)}$ by Theorem 6.3. Hence, Proposition 7.1 implies that the reduced $\mathrm{L}^{2}$-signature of $\left(W_{J}, \Gamma_{n}\right)$ and $\left(W_{-J}, \Gamma_{n}\right)$ are equal. It follows from Proposition 7.5 that $\int_{\omega \in S^{1}} \sigma_{\omega}(J) d \omega=\int_{\omega \in S^{1}} \sigma_{\omega}(-J) d \omega$. However, for all $\omega \in \mathbb{C}, \sigma_{\omega}(-J)=-\sigma_{\omega}(J)$. By assumption $\int_{\omega \in S^{1}} \sigma_{\omega}(J) d \omega \neq 0$. Therefore we have reached a contradiction. Hence $\mathscr{B} \ell_{n}(K(\eta, J)) \nsucceq \mathscr{B} \ell_{n}(K(\eta,-J))$. 


\section{Example}

Since the trefoil is a fibered knot ([Ro], p. 327), we can use the left-handed trefoil for $K$. Furthermore, since the integral of the Levine-Tristram signatures of the left-handed trefoil, integrated over the circle of unit length, is $\frac{4}{3}$, we can also use the left-handed trefoil for $J$. Finally, since the trefoil is equivalent to its reverse, it follows that $-J$ is the mirror-image of $J$. That is, $-J$ is the right-handed trefoil. Finally, we must choose $\eta$ so that $\eta \in \pi_{1}(E(K))^{(n)}$ but $\eta \notin \pi_{1}(E(K))^{(n+1)}$. For the case when $n=1$, it suffices to choose $\eta$ to be a curve which clasps a band of the standard Seifert surface. Therefore, we have the following construction.

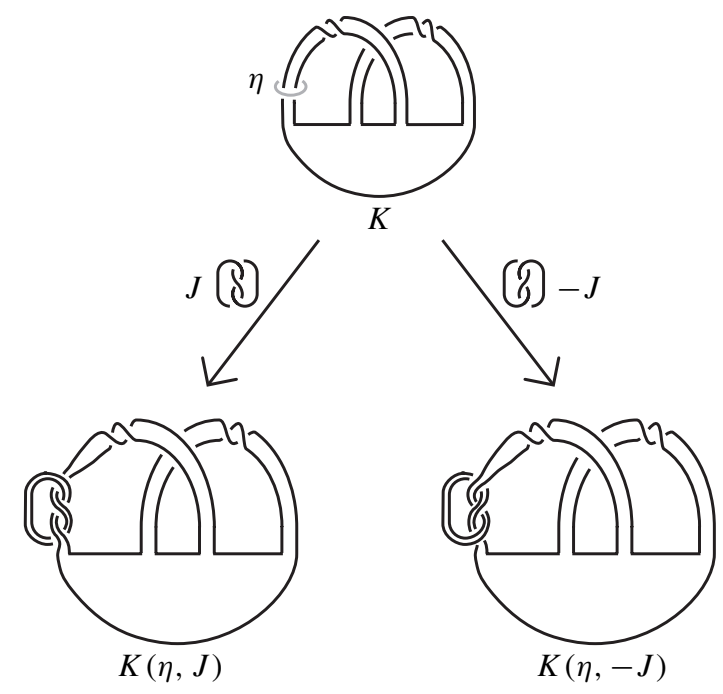

\section{References}

[C] T. Cochran, Noncommutative Knot Theory. Algebr. Geom. Topol. 4 (2004), 347-398. Zbl 1063.57011 MR 2077670

[COT1] T. Cochran, K. Orr, and P. Teichner, Knot Concordance, Whitney towers and L ${ }^{2}$-signature. Ann. of Math. 157 (2003), 433-519. Zbl 1044.57001 MR 1973052

[COT2] T. Cochran, K. Orr, and P. Teichner, Structure in the Classical Knot Concordance Group. Comment. Math. Helv. 79 (2004), 105-123. Zbl 1061.57008 MR 2031301

[Co] P. M. Cohn, Skew Fields. Encyclopedia Math. Appl. 57, Cambridge University Press, Cambridge 1995. Zbl 0840.16001 MR 1349108

[DK] J. Davis and P. Kirk, Lecture Notes in Algebraic Topology. Grad. Stud. in Math. 35, Amer. Math. Soc., Providence, RI, 2001. Zbl 1018.55001 MR 1841974 
[D] J. Duval, Forme de Blanchfield et cobordisme d'entrelacs bords. Comment. Math. Helv. 61 (1986), 617-635. Zbl 0632.57014 MR 0870709

[G] C. Gordon, Some aspects of classical knot theory. In Knot theory (Proc. Sem., Planssur-Bex, 1977), Lecture Notes in Math. 685, Springer-Verlag, Berlin 1978, 1-60. Zbl 0386.57002 MR 0521730

[HS] P. J. Hilton and U. Stammbach, A Course in Homological Algebra. Grad. Texts in Math. 4, Springer-Verlag, New York 1997. Zbl 0863.18001 MR 1438546

[Le] J. P. Levine, Knot Modules, I. Trans. Amer. Math. Soc. 229 (1977), 1-50. Zbl 0653.57012 MR 0461518

[Li] W. B. R. Lickorish, An Introduction to Knot Theory. Grad. Texts in Math. 175, SpringerVerlag, New York 1997. Zbl 0886.57001 MR 1472978

[K] C. Kearton, Blanchfield Duality and Simple Knots. Trans. Amer. Math. Soc. 202 (1975), 141-160. Zbl 0305.57016 MR 0358796

[MR] J. C. McConnell and J. C. Robson, Noncommutative Noetherian Rings. Grad. Stud. in Math. 30, Amer. Math. Soc., Providence, RI, 2001. Zbl 0980.16019 MR 1811901

[P] D. Passman, The Algebraic Structure of Group Rings. Pure and Applied Mathematics, John Wiley and Sons, New York 1977. Zbl 0368.16003 MR 0470211

[R] A. Ranicki, Exact Sequences in the Algebraic Theory of Surgery. Math. Notes 26, Princeton University Press, Princeton, NJ; University of Tokyo Press, Tokyo 1981. Zbl 0471.57012 MR 0620795

[Ro] D. Rolfsen, Knots and Links. Math. Lecture Ser. 7, Publish or Perish, Inc., Houston, TX, 1976. Zbl 0339.55004 MR 0515288

[Ste] B. Stenström, Rings of Quotients. Grundlehren Math. Wiss. 217, Springer-Verlag, New York 1975. Zbl 0296.16001 MR 0389953

[T] H. F. Trotter, On S-equivalence of Seifert Matrices. Invent. Math. 20 (1973), 173-207. Zbl 0269.15009 MR 0645546

Received June 28, 2004; revised August 12, 2005

Constance Leidy, Department of Mathematics, University of Pennsylvania, David Rittenhouse Laboratory, 209 South 33rd Street, Philadelphia, PA 19104-6395, U.S.A.

E-mail: cleidy@math.upenn.edu 